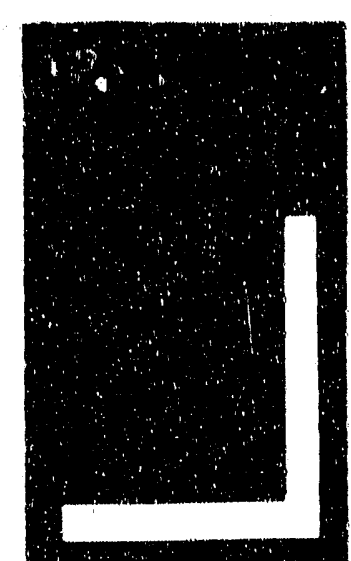

\title{
DCE/REF:
}

$\mathrm{DOE} / \mathrm{PC} / 79903-\cdots \mathrm{T} .4$

DE92 041182

\section{OPTICAL PROPERTIES OF FLYASH}

Contract NO. DE-AC22-87PC 79903

Quarterly Report for Perlod I April - 30 dune 1990

Prepared for Plttsburgh Energy Technology Center

Principal Investigator Professor S. A. Self

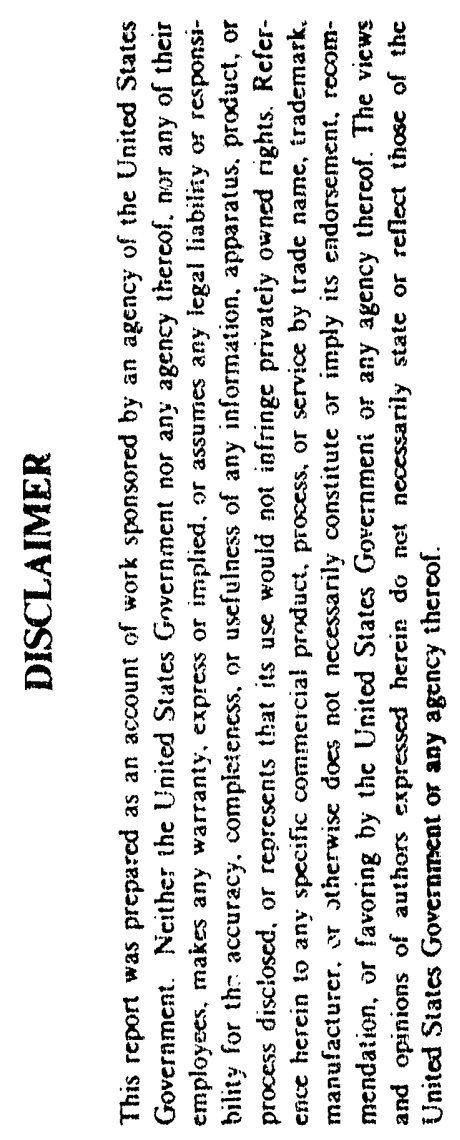

July 1990

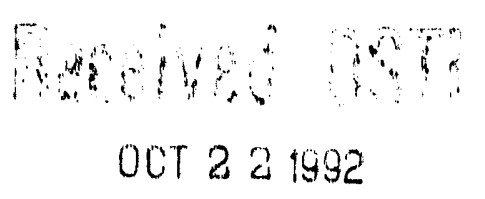

HIGH TEMPERATURE GASDYNAMICS LABORATORY

Mechanical Engineering Department

Stanford University

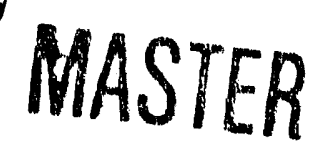




\section{OPTICAL PROPERTIES OF FLYASH \\ Contract No. DE-AC22-87PC 79903 \\ Quarterly Report for Period 1 April - 30 June 1990 \\ Prepared for Pittsburgh Energy Technology Center \\ Principal Investigator Professor S. A. Self}

\section{EXECUTIVE SUMMARY}

The general aims of this research are to provide a fundamental scientific basis for the physical understanding and reliable calculation of radiative heat transfer in coal combustion systems, particularly as it is influenced by the presence of inorganic constituents deriving from the mineral matter in coal.

The work is organized under four tasks. Tasks I and II were initiated in October 1987; Tasks III and IV were funded from October 1988.

Task 1. Charicterization of Flyash: Under this heading the chemical composition and size distribution of representative flyashes are being measured by appropriate microanalytical techniques to provide information required in Task 2.

Tusk 2. Measurements of the Optical Constants of Slags: Under this heading measurements of the infrared optical constants (i.e., the complex refractive index $m=n-i k$ ) of synthetic slags are being made as a function of wavelength and temperature for controlled compositions. Particular attention will be given to the contribution of the $\mathrm{Fe}_{2} \mathrm{O}_{3}$ content and its valence state. The data is being reduced to yield tormulae giving the complex refractive index over relevant ranges of wavelength and temperature, as a function of the relevant metal oxide constituents.

Task 3. Sample Calculations of the Radiant Properties of Flyash Dispersions: This component comprises various calculations to guide and evaluate the experimental work under the other three tasks.

Task 4. Measurement of the Radiant Properties of Flyash Dispersions: This benchscale experiment is planned to compare the measured radiant properties of a dispersion of well-characterized ash with computations based on data developed under the first two tasks.

In this eleventh quarter good progress has been made in all four arvas, as reported in the Quarterly Report, and summarized below. 


\section{Task 1}

The ashes being characterized are samples from power plants or pilot-scale combustors derived from combustion of six of the coals selected for study under the parallel PETC program on "Transformation of Inorganic Coal Constituents in Combustion Systems."

The principal features requiring characterization are particle size and composition distributions, including correlations between size and composition. Size distributions are being measured in house vy Coulter counter. Size and composition distributions are being determined by autusanted SEM/microprobe analysis at UNDERC. Other in-house characterization work under way includes size classification by wet sieving combined with classification by density using flotation/sedimentation techniques, low temperature ashing for the char content and magnetic separation for the magnetite content resulting from combustion of pyrite.

Size distributions for all six ashes over the range $0.5-60 \mu \mathrm{m}$ have been completed using the Coulter multisizer with a technique employing two orifices to cover the whole range. A suitable technique for matching distributions using two orifice sizes was devised. The measured distribution was found to be very well represented by truncated log normal distributions. Comparison with the size distribution from automated SEM shows some discrepancy which is still being investigated.

Micrographs of the ash samples prepared by UNDERC for automated SEM/EDX sizecomposition analysis revealed many agglomerates, casting some doubt on the validity of the data. A freeze-drying technique for sample preparation has been developed at Stanford which gives very well dispersed samples. The automated SEM/EDX analysis has been repeated using such samples and the restlts have been analyzed using statistical computer codes developed at Stanford. Ternary diagrams of the composition distributions (in color) have been prepared using software developed at Stanford. In the past quarter, automated SEM/EDX analyses have been completed and analysis of the data made for all six ashes.

In addition, density classification results have been obtained for all six ashes and compared with results from SEM analysis for composition, which, of course, determines the density.

\section{Task 2}

Methods for determining the infrared optical properties of solid synthetic and natural slags at low temperatures have been established in prior work at Stanford. In the present work the main effort has been devoted to the development of suitable apparatus and techniques for performing similar measurements on slags at temperatures to $2000 \mathrm{~K}$, when 
the slag is liquid. Basic experimental strategies have been decided and apparatus has been designed to accomplish this task. Preliminary tests at high temperatures during past quarters have resulted in the first reliable measurements of the infrared absor_tion of liquid slag.

Two complementary techniques involving infrared optical measurements on liquid samples of synthetic slag mainiained in an electric furnace have been developed. The first, for the wavelength range $1-5 \mu \mathrm{m}$ where the absorption index is low $\left(\mathrm{k} \leq 10^{-2}\right)$ employs a submerged platinum mirror to measure the absorption of thin films of slag by a double-pass technique. The second, applicable over the whole wavelength range $(1-12 \mu \mathrm{m})$, measures the surface reflectance of the liquid slag relative to that of a cold gold mirror in an external reference path.

The first technique has been successfully used to obtain the first reliable measurements of the infrared absorption of liquid slag (at $2000 \mathrm{~K}$ ).

Efforts have since been concentrated on developing and testing the second technique (for surface reflectivity measurements). It was deternined that the quality of the data obtained is limited by the differing absorptions due to $\mathrm{CO}_{2}$ and $\mathrm{H}_{2} \mathrm{O}$ in the hot measurement path and the cold reference path. To eliminate this problem, the whole apparatus has been enclosed in a chamber, purged with dry nitrogen.

With this modification, good measurements of the reflectivity of synthetic slag containing $5 \% \mathrm{Fe}$ at $1600^{\circ} \mathrm{C}$ were made over the whole wavelength range $1-12 \mu \mathrm{r}$. The data in the range 8-12 $\mu \mathrm{m}$ were reduced, using the Kramers-Kronig technique to give both the real and imaginary parts of the complex refrartive index.

Possible sources of uncertainty in these measurements have been critically investigated and resolved. In particular, a problem due to contamination of a mirror by furnace gases has been identified and steps taken to eliminate ; by redesign of the optical system. Also, in this quarter, reflectance measurements on liqui- slags containing zero, 1\%, 5\%,10\% and $20 \%$ iron (as $\mathrm{Fe}_{2} \mathrm{O}_{3}$ ) have been completed. The results have been reduced, using Kramers-Kronig analysis to yield the real (n) and imaginary (k) parts of the complex refractive index.

\section{Task 3}

Programs have been written for Mie scattering calculations which are then convolved with input on the size and optical constants distributions for a particulate dispersion to yield the spectral scattering and absorption coefficients of the aerosol. Additionally, a program has been written to solve the radiation transfer problem for a hornogeneous slab, utilizing the exact solution method of Case's normal modes. Input for the spectral scattering and 
absorption coefficients frorn the first program allows the spectral scattering, absorption and emission properties of the slab to be computed. These can then be integrcted over wavelength to yield the total radiative heat transfer characteristics of the slab.

These programs have been used to determine the importance of certain features of typical ashes for radiation transfer. These include the sensitivity of the optical/radiative properties of a flyash dispersion to (i) composition-size correlation, especially with regard to the distribution of iron oxides with particle size, and (ii) the presence of bubbles in the glassy ash particles.

This computational capability is also being used to evaluate the experimental conditions in the design of the apparatus for Task 4.

\section{Task 4}

Careful consideration has been given to the feasibility of various basic approaches for implementing the goals of this task. After evaluating various experimental techniques, a basic approach has been identified, which involves extinction measurements on flyash dispersed in suitable organic liquids. Measurements of the infrared transmission of three selected liquids have been made which confirm their suitability for this purpose. CaF2 windows for an absorption cell have been acquired and preliminary tests of a suitable cell design have : en made. 


\subsection{INTRODUCTION}

This is the eleventh quarterly report under DOE contract No. DE-AC22-87PC 79903 entitled "Optical Properties of Flyash." Tasks 1 and 2 of this program were funded from 15 September 1987. Tasks 3 and 4 were funded from 15 September 1988.

The general aims of this research are to provide a fundamental scientific basis for the physical understanding and reliable calculation of radiative heat transfer in coal combustion systems, particularly as it is influenced by the presence of inorganic constituents deriving from the mineral matter in coal. Some preliminary work in this area has been carried out at Stanford in the past several years with NSF support. The present program will greatly enlarge the scope of this work.

The complete, integrated program of theoretical and experimental work comprises four separate tasks.

Task 1. Characterization of Flyash

Task 2. Measurements of the Optical Constants of Slags

Task 3. Sample Calculations of the Radiant Properties of Flyash Dispersions.

Task 4. Measurements of the Radiative Properties of Flyash Dispersions.

In Task 1, the chemical composition and size distribution of representative flyashes are being measured by appropriate microanalytical techniques to provide information required in Tasks 2 and 3.

In "Task 2, measurements of the infrared optical constarts (i.e., the complex refractive index $\mathrm{m}=\mathrm{n}-\mathrm{ik}$ ) of synthetic slags are being made as a function of wavelength and temperature for controlled compositions. Particular attention is being given to the contribution of $\mathrm{Fe}_{2} \mathrm{O}_{3}$ content and its valence state. The data will be reduced to yield formulae giving the complex refractive index over relevant ranges of wavelength and temperature, as a function of the relevant metal oxide constituents.

In Task 3, sample calculations are being made for typical ash loadings, size distributions and compositions for simple geometries, with two main purposes: first, to provide insight and physical understanding of the role of flyash in radiative heat transfer in combustion systems; second, to indicate the sensitivity of the results to the characteristics of the input data. Such calculations will also be used to determine appropriate conditions and to predict the expected measured radiative properties for the experiment of Task 4. 
The experiment of Task 4 is designed to critically test our ability to predict the measured spectral emittance and scattering coefficient of flyash dispersions under wellcontrolled laboratory conditions utilizing the optical property data developed in Task 2. Particular attention will be paid to assessing the contribution of the char component in typical ashes. Any discrepancies between calculated and measured quantities revealed by these tests will be resolved by appropriate further studies.

A more detailed description of the scope of these tasks is given below. First, however, an outline is given of the rationale for the overall approach adopted in this program.

\subsection{Rationale of Overall Approach}

To account for the effects of flyash in radiative heat transfer calculations requires a knowledge of the contributions of the ash to the spectral absorption $\left(a_{\lambda}\right)$ and scattering $\left(\sigma_{\lambda}\right)$ coefficients of the particulate dispersion, together with the phase function $\Phi_{\lambda}$ describing the anisotropy of the scattering. These quantities depend on the particulate loading as well as the distributions of the size and optical properties of the particles.

For a spherical particle of homogeneous, optically isotropic material, characterized by a complex refractive index $m \equiv(n-i k)$ Mie theory allows one to compute the spectral absorption $\left(Q_{\lambda, \mathrm{a}}\right)$ and scattering $\left(Q_{\lambda, s}\right)$ efficiencies of the particle, as well as the phase function $\phi_{\lambda}$. For randomly polarized radiation, these quantities are a function of the particle size parameter $x \equiv(\pi \mathrm{d} / \lambda)$, and the complex refractive index $m(C, \lambda, T)$, a function of composition, wavelength and temperature.

For a monodispersion of identical spherical particles, of specified loading (i.e. number density), the particulate's contribution to the optical properties ( $a_{\lambda}, \sigma_{\lambda}$ and $\Phi_{\lambda}$ ) of the medium are simply related to the spectral properties $\left(Q_{\lambda, 2}, Q_{\lambda_{1},}, Q_{\lambda}\right)$ of a single particle. It is also straightforward to compute the spectral optical properties of the medium for a polydispersion of spheres of identical composition, by convolving the results of Mie calculations for spheres of varying diameter (i.e. $x$ ) for fixed wavelength (and hence fixed $m$ ), with the particle size distribution (assumed given). In the case of a particulate material, like flyash, for which it is reasonable to assume that individual particles are of homogeneous composition but the composition varies from particle to particle, it is still possible to compute the spectral characteristics of the particulate dispersion by dividing the particles into an appropriate number of classes of varying composition (and hence $m$ ), each having a specified size distribution, and summing over particle classes. 
In radiative heat transfer calculations, the contribution of the gas to the spectral absorption coefficient is added to that of the particles to obtain the combined optical properties of the medium on a spectral basis. These optical properties are then used as input-for a radiation transfer code to calculate radiative fluxes, on a spectral basis, for a particular combustor geometry and boundary conditions. Finally, to obtain total heat transfer quantities such as the overall radiant heat flux, integrations over wavelength must be made.

The procedure, outlined above, represents the only logical approach to the computation of radiative heat transfer in flyash laden combustion gases. To implement this proceciure requires, as input, a detailed characterization of the ash with respect to its size and (complex) refractive index distributions on a spectral basis.

Now, while techniques are available for determining the size distribution of powder samples, such as flyash, there are no practical means available for reliably determining the complex refractive index distribution of a complex material such as flyash either on a single particle basis, as a powder or as a dispersed aerosol. However, it is possible, using modern microanalytical techniques, specifically computer-automated SEM/EDX analysis, to determine the size and chemical composition of a heterogeneous powder on a particle by particle basis for a statistically large number of particles.

If the compositions of individual particles can be related to the complex refractive index of their material, then the characterization of a particular ash in terms of its size and composition distributions can lead to the necessary input for carrying out the calculations, cutlined above, to compute radiation transfer in combustion systems containing that ash.

Thus the key requirement, necessary for the implementation of this approach, is data on the optical constants (i.e. the components $n, k$ of the complex refractive index) as a function of composition, wavelength and temperature covering the range of compositions found in representative ashes. Since, as noted above, and emphasized in texts on the optical properties of particulate matter, it is impractical to extract reliable data on the optical constants of material in particulate form, the only viable approach is to make measurements on homogeneous bulk samples for which well-established techniques are available.

The foregoing arguments provide the rationale for the present program. Characterization of representative flyashes concerning their size and composition distributions constitutes Task 1, while measurements of the optical constants on bulk samples of synthetic slags as a function of relevant ranges of composition, wavelength and temperature constitute Task 2. Task 3 is designed to provide computational capabilities to support the other tasks, while Task 4 is planned to provide an experimental test that the measured optical properties of a dispersion of flyash can indeed be computed reliably from a knowledge of the size and composition distributions of the ash. 


\subsection{Description of Tasks}

\section{TASK 1 - Characterization of Flyash}

Extensive prior analyses of flyash from a wide range of coals plus analyses of the mineral matter in raw coals, together with knowledge of the transformation processes occurring duxing combustion, lead to the following overall picture of the nature of flyash.

The particle size distribution is very broad with a volume (or mass) mean diameter on the order of $10 \mu \mathrm{m}$. Typically it is well represented by a log normal distribution with the $1 \%$ and $99 \%$ sizes in a cumulative plot by volume occurring at $\sim 1 \mu \mathrm{m}$ and $70 \mu \mathrm{m}$ respectively. Evidence of a distinct submicron fume due to homogeneous condensation of volatile mineral matter is sometimes found, but this fraction can be expected to contribute negligibly to radiation transfer.

With regard to chemical composition, several distinct classes of particle can be identified and plausibly related to their origin and formation mechanisms.

By far the preponderant class, usually representing on the order of $90 \%$ or more of the ash on a mass basis, consists of vitreous (amorphous) material composed primarily of $\mathrm{SiO}_{2}$, $\mathrm{Al}_{2} \mathrm{O}_{3}, \mathrm{CaO}$ and $\mathrm{MgO}$, usually in that order, but containing varying smaller percentages of other metal oxides, notably $\mathrm{Fe}_{2} \mathrm{O}_{3}$. It can appropriately be identified as particles of impure (calcium) aluminosilicate glass derived from the microscopic clay-like mineral inclusions in the coal matrix. As char burnout proceeds these inclusions melt and sorm liquid globules on the surface of the char (which they do not wet) and are then released into the gas.

These glassy particles tend to be quite spherical with smooth surfaces and of reasonably homogeneous composition as is to be expected from their formation as liquid droplets. As they cool after release from the char surface, they remain in the vitreous state because the cooling rate is much faster than the crystallization rate for the formation of specific phases. The fact that the bulk of most ashes consists of reasonably spherical, homogeneous and vitreous (and therefore optically isotropic) particles is a very fortunate fact, since they satisfy the assumptions of the Mie theory remarkably well.

Micrographs of optically polished sections of ash cast in epoxy resin, shows that these glassy particles sometimes contain a number of small bubbles of gas evolved from the char and trapped in the particles as they iorm on the char surface. More rarely, large, thin-walled cenospheres are observed which presumably are "glass-blown" when a liquid drop covars a pore in the char from which a relatively large volume of gas is evolved under pressure. Although such cenospheres are very prominent ubjects in micrographs, their number is usually too small to significantly affect radiation transfer. 
Auger spectroscopic studies of ash often show a thin surface layer composed of volatile metals and high in sulfur (as sulfates) and water. The presence of a thin layer of adsorbed water containing sulfate ions controls the electrical resistance of the surface which is very important in the performance of electrostatic precipitators. However, this surface layer, of different composition from the underlying particle, is too thin $(\leq 100 \AA)$ relative to wavelengths of interest to affect the optical properties of the particle.

Apart from this major class of glassy particles, several distinct minor classes of particle types can be identified, each comprising, at most, a few percent by mass of the flyash. One such class consists of incompletely burned char particles which are clearly identified in optical and SEM microgrc.phs by the fact that they are black, of irregular shape and porous. The mass fraction of char depends on the particular coal and the combustor configuration and operating conditions. In modern combustors the mass fraction of unburned char is normally a few percent at most.

Another minor class consists of particles of adventitious incombustible mineral matter (e.g. quartz) which is contained in the pulverized coal feed. Such particles are usually large and of irregular shape, often showing rounded edges indicating partial melting.

A third minor class consists of magnetite $\left(\mathrm{Fe}_{3} \mathrm{O}_{4}\right)$ which derives from the combustion of pyrite $\left(\mathrm{FeS}_{2}\right)$ particles contained in the coal grind. These magnetite particles are black, generally spherical, magnetic and much denser than the glassy particles. The proportion of magnetite particles depends on the coal type, being largest in high sulfur coals, because the sulfur is mostly assiciated with pyrite. Recent work has shown that much of the pyrite in the coal grind can be removed by washing/sedimentation with a reduction in $\mathrm{SO}_{\mathrm{x}}$ emissions as high as $50 \%$ in some high sulfur coals.

The ashes selected for characterization are samples from power plants or pilot-scale combustors derived from the same seven coals selected for study under the parallel PETC program on "Transformation of Inorganic Coal Constituents in Combustion Systems" which comprise four bituminous, one sub-bituminous and two lignite coals.

A variety of techniques are being used in the characterization of these ashes. The principal method for determining size distributions employs a Coulter Multisizer which is sapable of giving accurate, reliable results of high resolution over a wide dynamic range $\leq 1 \mu \mathrm{m}$ to $\geq 100 \mu \mathrm{m}$.

Automated, computer-controlled, combired SEM/EDX microanalysis will be the principal technique used to determine the distributions of composition and size for a large number $(-1000)$ of particles for each ash. The size distributions will be compared with those obtained by the Coulter counter. 
Other techniques to be used include classification by density using liquids of varying density in a centrifuge, together with classification by size using a wet-sieving method. These techniques can yield density and size separated fractions for further examination by microanalytical techniques such as energy-dispersive X-ray spectroscopy. In addition, the magnetite particles may be separated by magnetic separation. The char content will be determined by low temperature ashing.

\section{TASK 2 - Measurements of Optical Constants of Synthetic Slags}

This task is planned to provide the basic optical properties data in a comprehensive and conveniently usable form. The optical constants (i.e., the components of the complex refractive index $m=n-i k$ ) of samples of synthetic slags of controlled compositions will be measured using established techniques involving transmission and surface reflectance methods. The wavelength range will extend from the visible to $12 \mu \mathrm{m}$, and the temperature range will extend to $2000 \mathrm{~K}$.

In earlier work at Stanford, supported by NSF, extensive measurements of this type were made on polished wafers of synthetic slags at temperatures up to $1200 \mathrm{~K}$. A major component of this task will be to extend such measurements to higher temperatures $(\sim 2000 \mathrm{~K})$ where the slag is liquid. This requires the development of modified techniques which present a number of more or less severe technical challenges.

Initially, the optical constants of the basic calcium-aluminosilicate host glass will be determined for the composition range defined by Task 1 . Subsequently, by adding infraredactive mineral oxide constituents in controlled amounts, one at a time, the modifications to $m(\lambda, T)$ produced by such constituents will be quantitatively determined. The particular constituents (and their range of mass fractions) to be examined will be determined by those disclosed by Task 1, taking account of knowledge of the optical activity at relevant wavelengths of such additions from the literature of glass technology. Specific constituents to be examined will include $\mathrm{Fe}_{2} \mathrm{O}_{3}$, taking especial account of its valence state ( $\mathrm{Fe}^{2+} / \mathrm{Fe}^{3+}$ ratio), and of $\mathrm{TiO}_{2}$. The contribution of the $\mathrm{OH}$ radical to the optical properties will be evaluated and quantified if significant.

The experimental data on $m(\lambda, T)$ as a function of composition, over the range relevant to coal ashes, will be reduced to generate simple correlation formulae. The latter will constitute the data base necessary to calculate the radiative properties of bulk slags and ash dispersions required for understanding and computing radiative transfer in coal combustion systems. 


\section{TASK 3-Sample Calculations of the Radiant Properties of Flyash Dispersions}

This task is intended to provide computational capabilities to support the other tasks. It includes the following components.

(i) A Mie scattering code to calculate the absorption and scattering efficiencies and phase function of a single sphere of specified size parameter and complex refractive index. A modified Mie code will also allow such computations for hollow spheres.

(ii) A code to convolve the results from (i) over a specified size distribution and loading to compute the absorption and scattering coefficients and phase function of a homogeneous polydispersion.

(iii) A code to sum the results of (ii) for a number of classes of particles of varying refractive indices and size distributions, i.e. for a heterogeneous polydispersion.

(iv) A radiation transfer code to calculate the absorption, scattering and emission characteristics of a homogenous, isothermal slab of dispersed ash on a spectral basis.

(v) A code to integrate the spectral results from (iv) over wavelength to yield the total radiative properties of the slab.

Calculations using these codes will be used to provide sensitivity analyses to guide the characterization work of Task 1, and to design and evaluate the results of Task 4.

\section{TASK 4 - Measurement of the Radiative Properties of Flyash Dispersions}

The purpose of this laboratory scale experiment is to test our ability to predict the measured radiative properties of a dispersion of well-characterized flyash. As such it will provide a critical test of the effectiveness of the overall approach adopted in this program. 


\subsection{PROGRESS IN THE PAST QUARTER}

\subsection{Task 1: Characterization of Fly Ash}

\subsubsection{Microprobe Study of Chemical Composition of Slag Samples:}

Chemical analysis of four slags, which were produced by melting the fly ashes, was performed using the electron microprobe at the Center for Material Research at Stanford. In sach case, the composition was determined at ten points scattered evenly over the sample surface. Because each sample is very homcgeneous, the crinpositions at these points showed little variation. The bulk composition was computed by averaging the data for the ten points. The results are presented in Table 7 and Table 8, and discussed in the next section.

\subsubsection{Microanalysis by SEM/EDX:}

The chemical composition data of the four fly ashes (Srn Miguel, Eagle Butte, Beulah and Upper Freeport) obtaines by C.CSEM (computer controlled scanning electron microanalysis) at UNDERC (University of North Dakota Energy Research Center) have been analyzed. We now have information of the chemical composition of one thousand or more particles selected randomly from a sample of each of the six fly ashes.

Temary plots are presented in Figure 1 to visually grasp the chemical composition distribution of the fly ashes with respect to the three major constituent elements: silicon, aluminum and iron. A color scheme has been used to indicate the variation of iron concentration. Clearly, each of the ashes is significantly different from the others. The San Miguel ash has a very low iron content. The centroid of the distribution of the Eagle Butte ash is shifted away from the $\mathrm{Al}$ and Si vertices and close to the 'Other' vertex. This is due to the high calcium content of the ash. This ash also contains a large number of particles with high silicon-zero aluminum content. Some of these are probably quartz particles. The distributions of the Upper Freeport and the San Miguel ashes are quite similar. The former is the only ash of the four with a significant number of particles with high iron content. It is interesting to note that there are very few particles with aluminum content $>50 \%$ and nonct that are entirely composed of silicon ard aluminum.

In Figures 2-4, the average chemical composition on an undersize basis are plotred. The elements shown are $\mathrm{Si}, \mathrm{Al}, \mathrm{Fe}$ and one or two other elernents that occur in abundance in a particular ash. The solid line shows the size distribution by count. In all cases, the compositional variation as particle diameter changes is small. In all ashes except Upfer Freepor, the silicon fraction increases gradually with particle diameter. The iron seems to be distributed evenly except for Illinois \#6, where it decreases with increasing particle dianeter, and in the case of Kentucky $\$$, where it shows some variation in the $1-10 \mu \mathrm{m}$ range. 

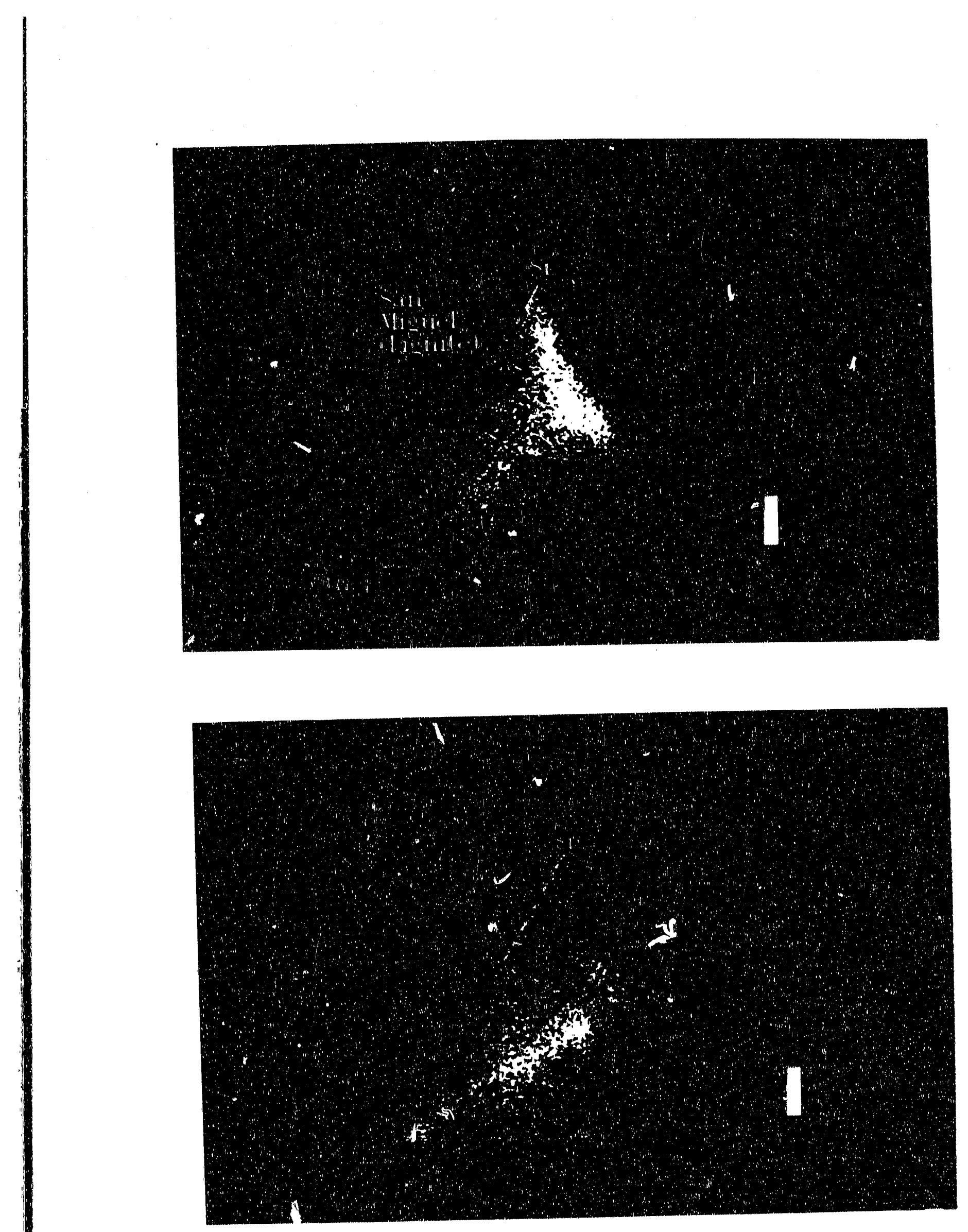

Figure 1: Ternary plots of fly ash chemical composition 

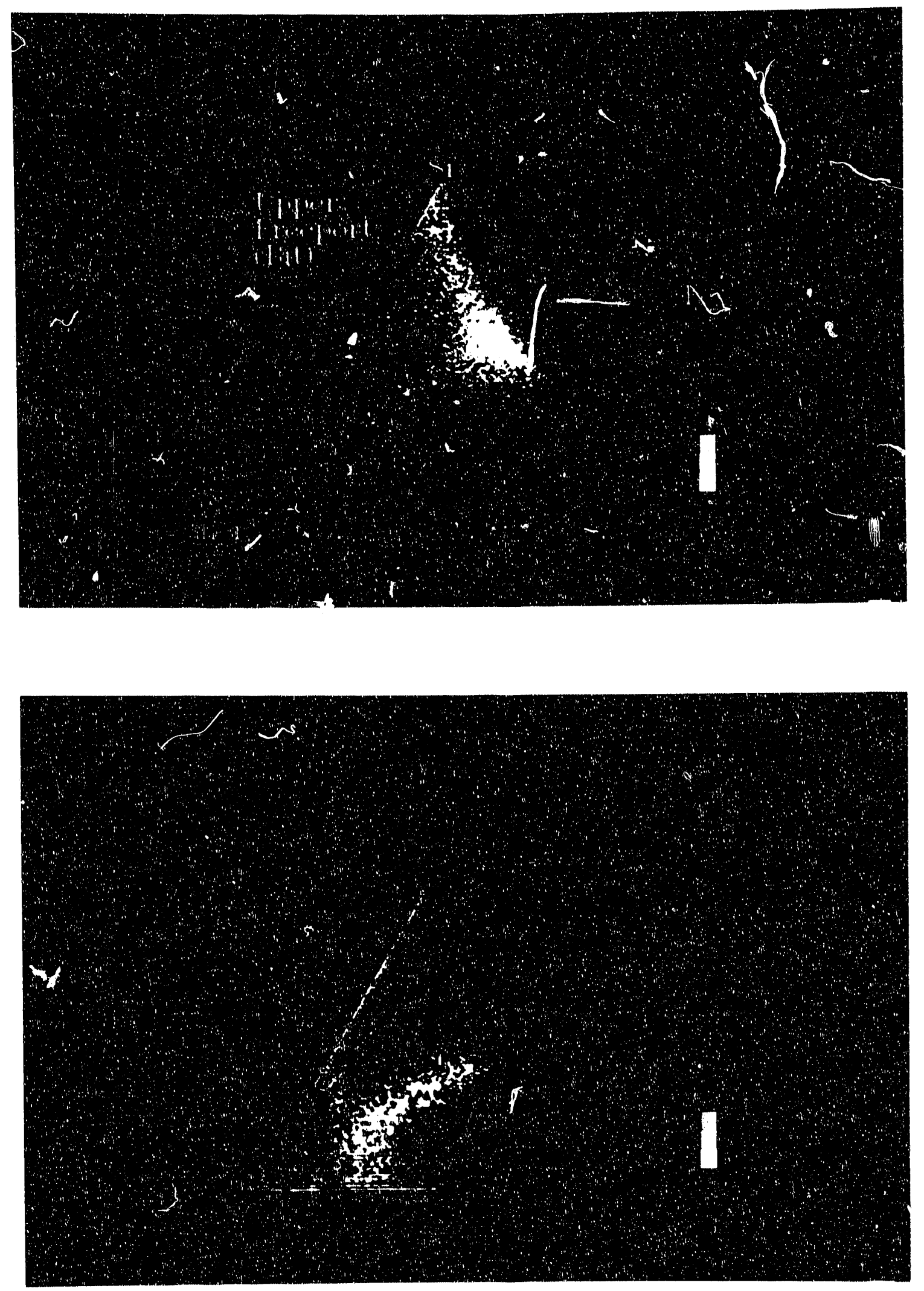

Figure 1 (continued) 

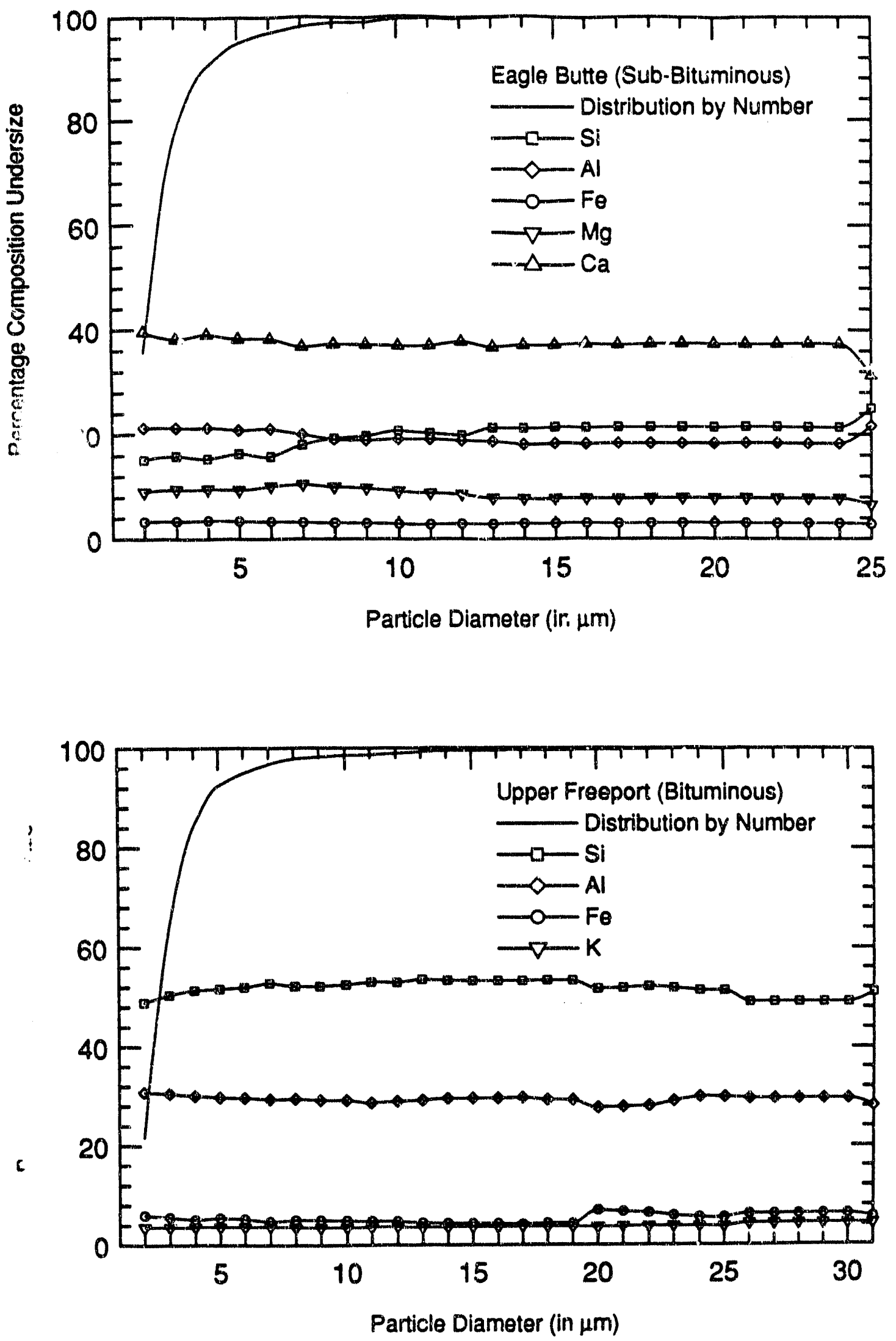

Fig 2: Average elemental composition of fly ash particles obtained from CCSEM/EDS 

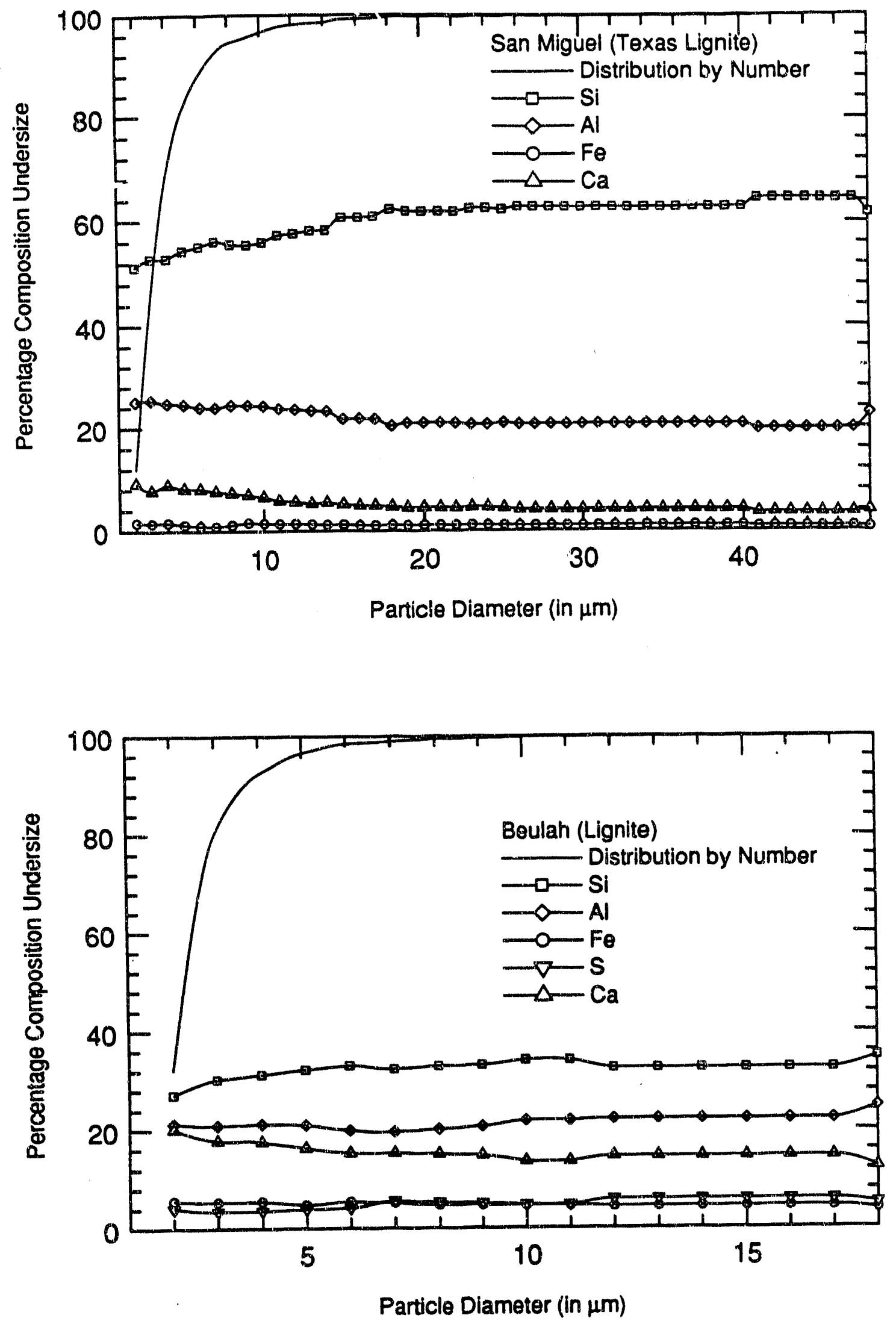

Figure 3: Average elemental composition of fly ash particles obtained from CCSEM 

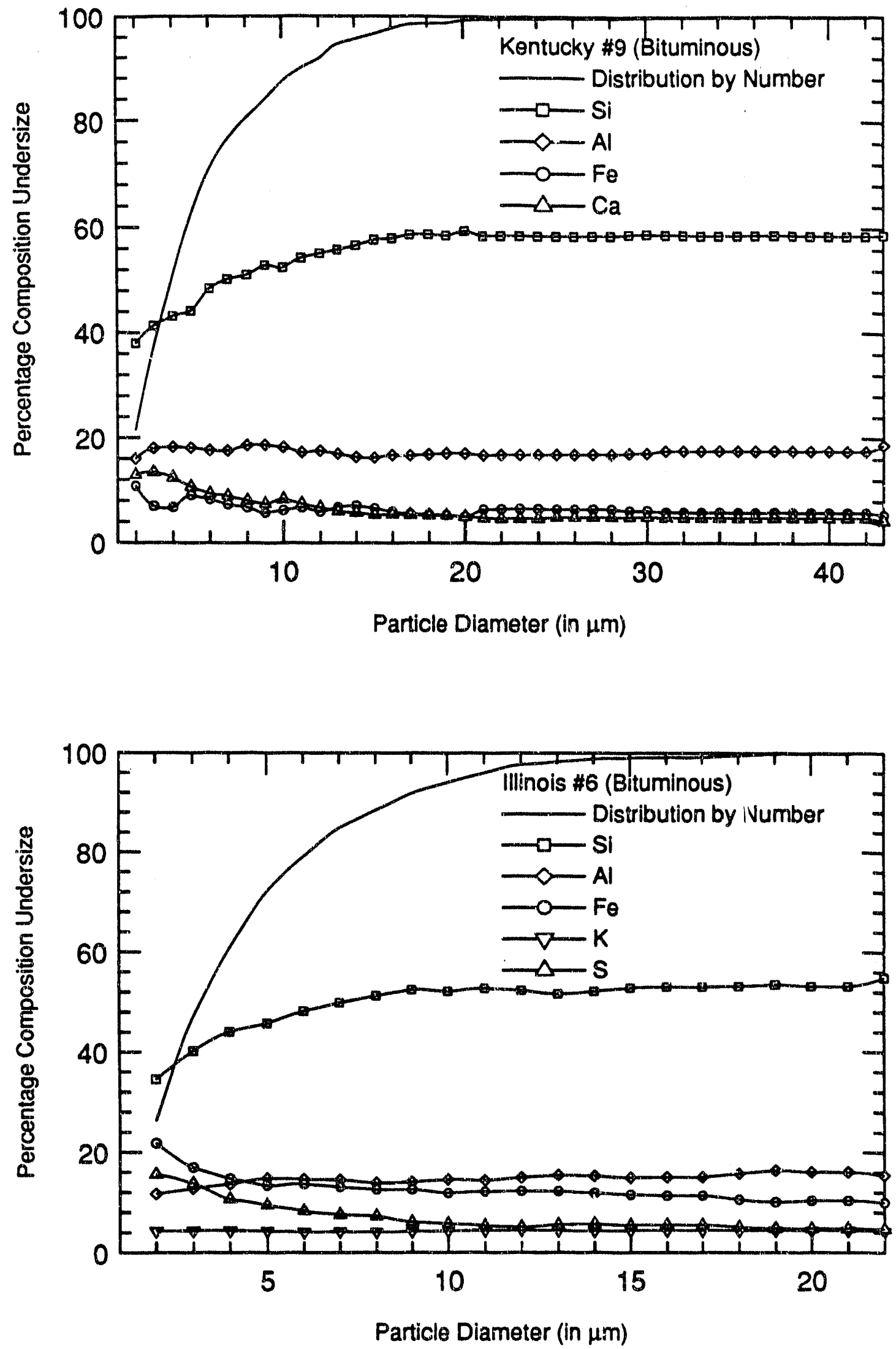

Figure 4: Average elemental composition of fly ash particles obtained from CCSEM/EDS 
The chemical composition as a function of particle diameter has been tabulated over increments of one micron after averaging the compositions of all particles in each range (Tables 1-6). The first column contains the particle diameter in microns. The number of particles sampled in each micron-size bin is shown in the second column. The rest of the columns show the oxide percentages corresponding to the twelve elements. For particle diameters $>6 \mu \mathrm{m}$, the accuracy of this composition distribution is limited by the small sample sizes (< 50 particles). The composition distributions will be converted to complex refractive index distributions using optical property data from Task 2.

In Table 7 and Table 8, the bulk compositions of the four ashes obtained by different means are compared. The CCSEM composition distributions (from Tabls 3 1-6) were combined with the size distribution obtained from the Coulter Multisizer for each of the fly ashes to obtain the bulk composition of column 1. The number of particles analyzed by CCSEM are shown in parentheses. The microprobe results on the melted ash are shown in the next column, and the last column shows the distribution obtained by PSI, Inc. (Reference 1). Considering the relatively small CCSEM sample size leads to a greater uncertainty, the results show reasonably good agreement with each other. Little or no sulfur was detected in the microprobe study because it escaped as vapor during slag preparation. The alumina content from the microprobe study is probably slightly high because a small amount of the alumina crucible dissolved during slag preparation. Still, the alumina content of the Beulah ash from the PSI study is very low. There is also a large difference between the iron content of the Kentucky \#9 as found by the microprobe analysis and the PSI study. These discrepancies could arise if the ashes analyzed were not identical because they arose from coals from different seams, or were produced under different combustion conditions. It is clear that the CCSEM study shows a consistently lower iron content for all the ashes. The reason is that the large magnetite particles that are high in iron content but are few in number were not detected. We are currently examining this data together with the density separation data in order to determine the iron content as accurately as possible.

The size distribution obtained from the CCSEM data has been compared with the datn from the Coulter Multisizer (see Figures 5-10). The difference between the two distributions are primarily due to the two reasons discussed below.

It is virtually impossible to prepare a fully deagglomerated ash sample for CCSEM. Our sample preparation technique leads to preparation of a relatively well deagglomerated sample, with few particle clusters per frame examined during CCSEM. These agglomerates are rejected by limiting the maximum acceptable shape factor to 1.1. However, these few ash particle clusters consist of numerous small ash particles $(1-5 \mu \mathrm{m})$ attached to one or two larger particles. This can easily be confirmed by examining transparent glass microspheres under an optical microscope. The fines can be seen to adhere to the sphere all over its surface. As a 
Table 1: Oxide composition of Illinois \#6 fly ash as a function of particle diameter.

\begin{tabular}{|c|c|c|c|c|c|c|c|c|c|c|c|c|c|}
\hline Dia. & o. & $\mathrm{Si}$ & $\mathbf{A l}$ & $\mathrm{Fe}$ & $\mathrm{Ca}$ & $\mathrm{Na}$ & $\mathbf{K}$ & $\mathrm{Ba}$ & $\mathrm{Ti}$ & $\mathbf{M g}$ & $\mathbf{P}$ & $\mathbf{S}$ & $\mathrm{Cl}$ \\
\hline 1 & & 39.1 & 12.1 & 18.9 & 2.5 & 0.2 & 2.8 & 0.8 & 1.4 & $0 ?$ & 0.6 & 20.8 & 0.3 \\
\hline 2 & & 0.0 & & 12.9 & 2.3 & 0.4 & 3.0 & 0.9 & 1.3 & 0.2 & 0.5 & 18.0 & 0.4 \\
\hline 3 & 142 & 53.0 & 14.4 & 10.9 & 3.8 & 0.1 & 2.9 & 0.9 & 1.5 & 0.2 & 0.4 & 11.6 & 0.3 \\
\hline 4 & & 53.8 & 15.9 & 10.1 & 3.1 & 0.2 & 2.7 & 0.7 & 1.2 & 0.2 & 0.3 & 11.5 & 0.3 \\
\hline 5 & 72 & 58.2 & 14.8 & 11.6 & 1.7 & 0.2 & 2.5 & 0.8 & 1.1 & 0.1 & 0.1 & 8.7 & 0.2 \\
\hline 6 & & 60.7 & 14.5 & 9.4 & 2.3 & 0.1 & 2.9 & 0.5 & 1.1 & 0.1 & 0.1 & 0.0 & 0.2 \\
\hline 7 & & 62.7 & 12.1 & 8.8 & 3.8 & 0.2 & 2.4 & 0.6 & 0.9 & 0.1 & 0.2 & 8.1 & 0.2 \\
\hline 8 & & 62.0 & 15.2 & 11.3 & 2.7 & 0.2 & 3.0 & 0.5 & 0.9 & 0.1 & 0.2 & 3.6 & 0.3 \\
\hline 9 & & 57.7 & 17.4 & 7.3 & 4.9 & 0.2 & 3.0 & 0.6 & 1.8 & 0.2 & 0.2 & 6.6 & 0.2 \\
\hline 10 & & 62.6 & 13.4 & 11.9 & 1.9 & 0.2 & 3.2 & 0.5 & 1.3 & 0.0 & 0.2 & 4.4 & 0.4 \\
\hline 11 & 11 & 57.9 & 19.1 & 10.5 & 1.8 & 0.3 & 3.1 & 0.8 & 0.8 & 0.3 & 0.1 & 5.4 & 0.1 \\
\hline 12 & 6 & 48.0 & 20.1 & 9.8 & 1.7 & 0.1 & 2.0 & 0.8 & 2.1 & 0.2 & 0.0 & 15.0 & 0.4 \\
\hline 13 & 6 & 65.1 & 13.4 & 5.8 & 0.6 & 0.2 & 2.4 & 0.6 & 0.8 & 0.0 & 1.0 & 9.6 & 0.5 \\
\hline 14 & 2 & 81.3 & 8.4 & 1.9 & 0.7 & 0.4 & 3.9 & 0.6 & 2.3 & 0.0 & 0.0 & 0.0 & 0.5 \\
\hline 15 & 1 & 69.3 & 0.1 & 2.3 & 0.0 & 0.0 & 2.6 & 1.8 & 2.7 & 0.0 & 0.0 & 1.3 & 0.0 \\
\hline 17 & 4 & 62.1 & 22.0 & 2.5 & 2.3 & 0.0 & 3.2 & 1.2 & 3.4 & 0.0 & 0.0 & 2.7 & 0.5 \\
\hline 18 & 3 & 65.8 & 22.0 & 3.1 & 0.8 & 0.2 & 3.0 & 1.0 & 0.6 & 0.0 & 0.0 & 2.7 & 0.9 \\
\hline 19 & 1 & 55.1 & 7.7 & 18.4 & 0.8 & 0.0 & 2.1 & 3.9 & 3.9 & 1.0 & 0.0 & 5.8 & 1.2 \\
\hline 21 & 1 & 96.0 & 0.0 & 0.0 & 0.0 & 0.0 & 0.0 & 0.0 & 1.6 & 0.0 & 0.0 & 2.4 & 0.0 \\
\hline
\end{tabular}


Table 2: Oxide composition of Kentucky \#9 fly ash as a function of particle diameter.

\begin{tabular}{|c|c|c|c|c|c|c|c|c|c|c|c|c|c|}
\hline Dia. & No. & $\mathrm{Si}$ & $\mathrm{Al}$ & $\mathrm{Fe}$ & $\mathrm{Ca}$ & $\mathrm{Na}$ & $\mathbf{K}$ & $\mathrm{Ba}$ & $\mathrm{Ti}$ & $\mathrm{Mg}$ & $\mathrm{P}$ & $\mathrm{S}$ & $\mathrm{Cl}$ \\
\hline 1 & 204 & 43.4 & 15.5 & 9.3 & 10.2 & 0.6 & 2.6 & 0.8 & 1.5 & 0.3 & 0.9 & 13.0 & 0.8 \\
\hline 2 & 151 & 50.1 & 19.3 & 5.1 & 10.7 & 0.5 & 2.7 & 1.1 & 1.6 & 0.3 & 0.3 & 7.3 & 0.9 \\
\hline 3 & 132 & 50.8 & 18.8 & 6.5 & 9.6 & 0.2 & 2.7 & 1.1 & 1.4 & 0.2 & 0.3 & 7.5 & 0.8 \\
\hline 4 & 104 & 52.2 & 18.7 & 10.3 & 7.6 & 0.2 & 2.6 & 1.0 & 1.7 & 0.2 & 0.3 & 4.8 & 0.4 \\
\hline 5 & 83 & 61.3 & 17.3 & 6.2 & 6.2 & 0.3 & 3.1 & 0.1 & 1.1 & 0.0 & 0.1 & 0.4 & 0.3 \\
\hline 6 & 52 & 61.7 & 17.9 & 4.5 & 7.4 & 0.3 & 2.5 & 0.9 & 1.6 & 0.1 & 0.1 & 2.9 & 0.3 \\
\hline 7 & 39 & 60.0 & 21.4 & 4.5 & 4.6 & 0.3 & 2.7 & 0.6 & 1.0 & 0.0 & 0.1 & 4.5 & 0.3 \\
\hline 8 & 32 & 67.0 & 19.3 & 1.9 & 4.1 & 0.3 & 2.9 & 0.6 & 0.7 & 0.1 & 0.0 & 2.9 & 0.1 \\
\hline 9 & 33 & 58.3 & 17.5 & 6.8 & 9.2 & 0.1 & 2.2 & 0.7 & 0.8 & 0.1 & 0.1 & 3.7 & 0.5 \\
\hline 10 & 24 & 68.1 & 13.4 & 8.8 & 3.1 & 0.3 & 3.0 & 0.7 & 0.8 & 0.1 & 0.0 & 1.6 & 0.2 \\
\hline 11 & 18 & 67.5 & 19.9 & 1.5 & 2.6 & 0.3 & 3.6 & 0.7 & 1.3 & 0.0 & 0.0 & 2.2 & 0.3 \\
\hline 12 & 26 & 65.3 & 14.9 & 8.5 & 2.9 & 0.3 & 2.8 & 0.6 & 1.5 & 0.1 & 0.0 & 2.6 & 0.5 \\
\hline 13 & 10 & 69.3 & 11.4 & 9.4 & 2.2 & 0.3 & 2.4 & 0.2 & 0.5 & 0.0 & 0.0 & 4.0 & 0.3 \\
\hline 14 & 9 & 74.0 & 15.2 & 1.7 & 1.3 & 0.3 & 4.9 & 0.1 & 0.9 & 0.0 & 0.1 & 1.3 & 0.2 \\
\hline 15 & 10 & 68.4 & 20.9 & 1.3 & 4.8 & 0.1 & 1.3 & 1.5 & 0.9 & 0.1 & 0.0 & 0.7 & 0.1 \\
\hline 16 & 8 & 72.1 & 16.3 & 1.5 & 4.0 & 0.4 & 3.5 & 0.4 & 1.0 & 0.0 & 0.0 & 0.7 & 0.1 \\
\hline 17 & 2 & 66.2 & 24.4 & 1.1 & 0.7 & 0.4 & 2.5 & 0.6 & 0.0 & 0.0 & 0.0 & 3.9 & 0.3 \\
\hline 18 & 1 & 56.7 & 27.5 & 0.0 & 3.6 & 0.0 & 3.1 & 1.2 & 0.0 & 0.0 & 0.0 & 7.8 & 0.0 \\
\hline 19 & 5 & 73.0 & 16.0 & 1.3 & 1.9 & 0.4 & 2.5 & 1.2 & 0.7 & 0.0 & 0.0 & 3.0 & 0.0 \\
\hline 20 & 2 & 38.7 & 8.7 & 40.1 & 0.9 & 0.5 & 2.8 & 0.0 & 0.0 & 0.0 & 0.0 & 7.3 & 0.9 \\
\hline 21 & 1 & 64.5 & 22.0 & 7.6 & 0.0 & 0.7 & 1.3 & 0.0 & 0.0 & 0.0 & 0.0 & 4.0 & 0.0 \\
\hline 24 & 1 & 68.1 & 18.1 & 1.5 & 8.2 & 0.0 & 1.9 & 0.6 & 0.0 & 0.0 & 0.0 & 0.0 & 1.6 \\
\hline 28 & 1 & 69.2 & 20.0 & 0.8 & 3.7 & 0.0 & 1.9 & 1.8 & 2.7 & 0.0 & 0.0 & 0.0 & 0.0 \\
\hline 30 & 1 & 65.5 & 25.9 & 0.0 & 2.2 & 0.0 & 4.5 & 0.6 & 0.0 & 0.0 & 0.0 & 1.3 & 0.0 \\
\hline 42 & 1 & 66.6 & 25.9 & 2.3 & 0.7 & 0.7 & 3.8 & 0.0 & 0.0 & 0.0 & 0.0 & 0.0 & 0.0 \\
\hline
\end{tabular}


Table 3: Oxide composition of Upper Freeport fly ash as a function of particle diameter.

\begin{tabular}{|c|c|c|c|c|c|c|c|c|c|c|c|c|c|}
\hline Dia. & No. & $\mathrm{Si}$ & $\mathrm{Al}$ & $\mathrm{Fe}$ & $\mathrm{Ca}$ & $\mathrm{Na}$ & $\mathrm{K}$ & $\mathrm{Ba}$ & $\mathrm{Ti}$ & $\mathrm{Mg}$ & $\mathrm{P}$ & $\mathrm{S}$ & $\mathrm{Cl}$ \\
\hline 1 & 384 & 55.2 & 31.4 & 5.2 & 1.3 & 0.5 & 2.3 & 0.6 & 1.0 & 0.4 & 0.4 & 1.3 & 0.3 \\
\hline 2 & 750 & 57.1 & 30.5 & 5.0 & 1.2 & 0.4 & 2.3 & 0.5 & 0.9 & 0.3 & 0.3 & 1.1 & 0.2 \\
\hline 3 & 364 & 59.0 & 29.8 & 4.0 & 1.0 & 0.4 & 2.4 & 0.6 & 0.8 & 0.4 & 0.3 & 1.0 & 0.3 \\
\hline 4 & 141 & 58.7 & 29.5 & 5.7 & 0.8 & 0.4 & 2.4 & 0.4 & 0.7 & 0.2 & 0.1 & 0.8 & 0.2 \\
\hline 5 & 45 & 60.4 & 29.3 & 3.3 & 0.8 & 0.3 & 2.4 & 0.4 & 0.6 & 0.7 & 0.1 & 1.6 & 0.1 \\
\hline 6 & 32 & 64.1 & 27.8 & 1.6 & 1.4 & 0.3 & 2.7 & 0.4 & 0.6 & 0.4 & 0.1 & 0.5 & 0.2 \\
\hline 7 & 19 & 54.9 & 31.0 & 5.2 & 1.9 & 0.4 & 1.8 & 1.1 & 1.4 & 0.6 & 0.5 & 0.8 & 0.4 \\
\hline 8 & 7 & 58.5 & 24.1 & 4.6 & 6.7 & 0.2 & 2.0 & 0.8 & 0.5 & 1.0 & 0.0 & 1.6 & 0.0 \\
\hline 9 & 6 & 62.5 & 29.2 & 2.7 & 0.4 & 0.0 & 2.4 & 0.9 & 1.6 & 0.1 & 0.0 & 0.2 & 0.0 \\
\hline 10 & 2 & 78.7 & 14.3 & 1.1 & 0.0 & 1.1 & 3.4 & 0.6 & 0.8 & 0.0 & 0.0 & 0.0 & 0.0 \\
\hline 11 & 5 & 59.8 & 31.3 & 2.9 & 0.7 & 0.6 & 2.5 & 0.5 & 0.4 & 0.0 & 0.0 & 1.1 & 0.3 \\
\hline 12 & 5 & 64.7 & 30.6 & 1.5 & 0.1 & 0.4 & 1.9 & 0.1 & 0.7 & 0.0 & 0.0 & 0.0 & 0.0 \\
\hline 13 & 4 & 57.5 & 33.8 & 3.2 & 0.0 & 0.2 & 2.9 & 0.7 & 0.7 & 0.0 & 0.0 & 1.0 & 0.0 \\
\hline 14 & 1 & 59.6 & 29.4 & 2.3 & 2.2 & 0.0 & 2.6 & 1.2 & 2.7 & 0.0 & 0.0 & 0.0 & 0.0 \\
\hline 16 & 1 & 60.1 & 32.1 & 0.0 & 0.0 & 0.0 & 3.2 & 0.6 & 0.0 & 0.0 & 0.0 & 4.0 & 0.0 \\
\hline 17 & 1 & 62.6 & 22.1 & 5.3 & 4.5 & 0.0 & 1.9 & 0.0 & 0.9 & 0.0 & 0.0 & 2.7 & 0.0 \\
\hline 19 & 1 & 37.3 & 4.9 & 44.3 & 4.5 & 1.7 & 2.3 & 4.3 & 0.0 & 0.0 & 0.0 & 0.0 & 0.6 \\
\hline 20 & 1 & 61.7 & 31.3 & 1.5 & 0.0 & 0.0 & 3.2 & 1.8 & 0.0 & 0.0 & 0.0 & 0.0 & 0.5 \\
\hline 21 & 1 & 64.0 & 30.8 & 1.5 & 0.0 & 0.0 & 2.5 & 1.2 & 0.0 & 0.0 & 0.0 & 0.0 & 0.0 \\
\hline 22 & 1 & 53.8 & 39.6 & 0.7 & 0.7 & 0.7 & 3.2 & 0.0 & 0.0 & 0.0 & 0.0 & 1.3 & 0.0 \\
\hline 23 & 1 & 52.7 & 39.4 & 0.8 & 0.0 & 0.7 & 1.9 & 1.8 & 2.7 & 0.0 & 0.0 & 0.0 & 0.0 \\
\hline 25 & 1 & 32.0 & 30.6 & 11.1 & 5.9 & 0.0 & 7.2 & 2.7 & 4.0 & 2.0 & 0.0 & 4.5 & 0.0 \\
\hline 30 & 1 & 72.6 & 18.0 & 1.5 & 0.0 & 0.0 & 3.2 & 2.4 & 1.8 & 0.0 & 0.0 & 0.0 & 0.5 \\
\hline
\end{tabular}


Table 4: Oxide composition of Eagle Butte fly ash as a function of particle diameter.

\begin{tabular}{|c|c|c|c|c|c|c|c|c|c|c|c|c|c|}
\hline Dia. & No. & $\mathrm{Si}$ & $\mathrm{Al}$ & $\mathrm{Fe}$ & $\mathrm{Ca}$ & $\mathrm{Na}$ & $\mathrm{K}$ & $\mathrm{Ba}$ & $\mathrm{Ti}$ & $\mathrm{Mg}$ & $\mathbf{P}$ & $\mathbf{S}$ & $\mathrm{Cl}$ \\
\hline 1 & 672 & 19.2 & 24.7 & 3.0 & 35.0 & 0.4 & 0.3 & 1.0 & 1.6 & 9.1 & 0.8 & 4.5 & 0.3 \\
\hline 2 & 782 & 19.8 & 25.3 & 3.2 & 33.6 & 0.6 & 0.3 & 0.9 & 1.5 & 10.2 & 0.7 & 3.6 & 0.3 \\
\hline 3 & 245 & 18.3 & 25.1 & 3.5 & 35.3 & 0.9 & 0.3 & 0.8 & 1.3 & 10.2 & 0.7 & 3.5 & 0.3 \\
\hline 4 & 91 & 23.8 & 23.5 & 2.7 & 32.7 & 1.6 & 0.3 & 0.6 & 1.2 & 9.5 & 0.6 & 3.2 & 0.4 \\
\hline 5 & 40 & 16.6 & 25.0 & 2.8 & 34.6 & 1.3 & 0.4 & .7 & 1.3 & 13.8 & 0.5 & 2.8 & 0.2 \\
\hline 6 & 21 & 36.9 & 16.4 & 1.9 & 24.9 & 1.4 & 0.1 & 0.7 & 1.4 & 13.1 & 0.1 & 2.6 & 0.4 \\
\hline 7 & 16 & 32.0 & 16.0 & 2.6 & 36.5 & 1.0 & 0.2 & 1.0 & 1.5 & 6.5 & 0.3 & 2.1 & 0.4 \\
\hline 8 & 3 & 41.6 & 18.8 & 0.9 & 27.6 & 1.5 & 0.5 & 0.9 & 2.7 & 4.4 & 0.0 & 1.0 & 0.2 \\
\hline 9 & 8 & 34.1 & 21.6 & 1.3 & 31.9 & 0.7 & 0.1 & 0.4 & 1.1 & 5.0 & 0.9 & 2.6 & 0.2 \\
\hline 10 & 3 & 14.6 & 23.4 & 1.7 & 30.7 & 1.8 & 0.0 & 1.7 & 1.8 & 2.3 & 0.0 & 22.1 & 0.0 \\
\hline 11 & 1 & 0.0 & 4.3 & 6.4 & 76.8 & 0.0 & 0.9 & 1.7 & 5.0 & 0.0 & 0.0 & 1.9 & 3.0 \\
\hline 12 & 3 & 45.5 & 20.9 & 2.7 & 20.6 & 3.6 & 0.2 & 0.0 & 0.7 & 0.3 & 0.0 & 5.6 & 0.0 \\
\hline 13 & 1 & 28.1 & 5.9 & 5.4 & 45.5 & 0.0 & 0.0 & 0.0 & 0.0 & 5.2 & 0.0 & 9.4 & 0.6 \\
\hline 24 & 1 & 49.3 & 38.4 & 1.6 & 2.3 & 6.7 & 0.7 & 0.0 & 0.0 & 0.0 & 0.0 & 0.0 & 1.1 \\
\hline
\end{tabular}

Table 5: Oxide composition of Beulah fly ash as a function of particle diameter.

\begin{tabular}{|c|c|c|c|c|c|c|c|c|c|c|c|c|c|}
\hline Dia. & No. & $\mathbf{S i}$ & $\mathbf{A l}$ & $\mathbf{F e}$ & $\mathbf{C a}$ & $\mathbf{N a}$ & $\mathbf{K}$ & $\mathbf{B a}$ & $\mathbf{T i}$ & $\mathbf{M g}$ & $\mathbf{P}$ & $\mathbf{S}$ & $\mathbf{C l}$ \\
\hline 1 & 357 & 33.4 & 23.4 & 4.6 & 17.2 & 4.5 & 0.8 & 1.0 & 1.4 & 6.5 & 0.5 & 5.9 & 0.9 \\
\hline 2 & 535 & 37.3 & 23.1 & 4.6 & 14.7 & 5.5 & 0.9 & 1.0 & 1.5 & 4.7 & 0.4 & 5.0 & 1.2 \\
\hline 3 & 134 & 38.9 & 24.0 & 4.9 & 14.2 & 4.8 & 1.2 & 0.7 & 1.0 & 3.9 & 0.4 & 5.5 & 0.6 \\
\hline 4 & 47 & 41.6 & 22.3 & 2.1 & 10.4 & 7.4 & 1.5 & 1.1 & 1.5 & 2.4 & 0.2 & 6.0 & 3.6 \\
\hline 5 & 21 & 44.2 & 14.6 & 9.0 & 8.3 & 4.6 & 2.4 & 0.5 & 0.8 & 3.5 & 0.3 & 7.3 & 4.6 \\
\hline 6 & 5 & 25.0 & 16.5 & 0.6 & 10.9 & 4.0 & 3.1 & 0.1 & 0.5 & 0.0 & 0.0 & 37.6 & 1.7 \\
\hline 7 & 6 & 44.6 & 24.9 & 0.5 & 11.9 & 1.4 & 1.4 & 1.0 & 0.8 & 10.3 & 0.0 & 2.8 & 0.4 \\
\hline 8 & 1 & 45.8 & 48.5 & 1.5 & 2.2 & 0.0 & 1.9 & 0.0 & 0.0 & 0.0 & 0.0 & 0.0 & 0.0 \\
\hline 9 & 3 & 53.2 & 36.4 & 2.8 & 0.0 & 0.7 & 2.8 & 0.6 & 1.8 & 0.3 & 0.0 & 1.4 & 0.0 \\
\hline 11 & 1 & 0.0 & 29.4 & 0.8 & 25.6 & 0.7 & 0.0 & 0.0 & 0.0 & 4.6 & 0.0 & 38.8 & 0.0 \\
\hline 17 & 1 & 53.6 & 38.3 & 0.8 & 0.7 & 0.0 & 3.9 & 0.0 & 0.9 & 0.0 & 0.0 & 1.3 & 0.5 \\
\hline
\end{tabular}


Table 6: Oxide composition of San Miguel fly ash as a function of particle diameter.

\begin{tabular}{|c|c|c|c|c|c|c|c|c|c|c|c|c|c|}
\hline Dia. & No. & $\mathrm{Si}$ & $\mathrm{Al}$ & $\mathrm{Fe}$ & $\mathrm{Ca}$ & $\mathrm{Na}$ & $\mathrm{K}$ & $\mathrm{Ba}$ & $\mathrm{Ti}$ & $\mathrm{Mg}$ & $\mathrm{P}$ & $\mathrm{S}$ & $\mathrm{Cl}$ \\
\hline 1 & 186 & 58.3 & 26.0 & 1.3 & 7.3 & 2.7 & 0.8 & 0.7 & 1.0 & 0.4 & 0.3 & 0.9 & 0.3 \\
\hline 2 & 519 & 60.0 & 26.1 & 1.4 & 6.0 & 2.7 & 0.8 & 0.6 & 0.9 & 0.3 & 0.1 & 0.8 & 0.2 \\
\hline 3 & 356 & 59.9 & 25.0 & 1.4 & 7.6 & 2.4 & 0.7 & 0.5 & 0.7 & 0.4 & 0.1 & 1.1 & 0.2 \\
\hline 4 & 197 & 63.3 & 24.8 & 0.7 & 5.7 & 2.6 & 0.7 & 0.4 & 0.8 & 0.1 & 0.1 & 0.5 & 0.2 \\
\hline 5 & 106 & 64.6 & 23.2 & 0.4 & 6.6 & 2.6 & 0.8 & 0.4 & 0.6 & 0.1 & 0.1 & 0.4 & 0.2 \\
\hline 6 & 68 & 65.9 & 23.8 & 0.3 & 5.0 & 2.5 & 0.8 & 0.4 & 0.5 & 0.1 & 0.0 & 0.5 & 0.1 \\
\hline 7 & 39 & 59.9 & 27.0 & 2.3 & 4.6 & 2.6 & 0.8 & 0.8 & 1.2 & 0.0 & 0.1 & 0.5 & 0.3 \\
\hline 8 & 16 & 62.0 & 24.6 & 4.8 & 2.3 & 2.5 & 0.9 & 0.6 & 1.3 & 0.3 & 0.0 & 0.5 & 0.2 \\
\hline 9 & 17 & 67.4 & 23.9 & 0.3 & 2.6 & 2.7 & 0.7 & 0.1 & 0.8 & 0.0 & 0.0 & 0.9 & 0.3 \\
\hline 10 & 16 & 72.8 & 20.7 & 0.6 & 1.0 & 2.7 & 1.2 & 0.2 & 0.4 & 0.1 & 0.0 & 0.4 & 0.1 \\
\hline 11 & 7 & 69.1 & 21.2 & 0.2 & 1.9 & 2.2 & 1.0 & 1.6 & 1.1 & 0.3 & 0.0 & 1.3 & 0.2 \\
\hline 12 & 5 & 75.2 & 19.0 & 0.0 & 0.4 & 2.4 & 1.1 & 0.5 & 0.3 & 0.0 & 0.0 & 0.5 & 0.5 \\
\hline 13 & 3 & 67.2 & 20.6 & 0.2 & 7.6 & 1.1 & 0.6 & 0.4 & 1.2 & 0.3 & 0.0 & 0.5 & 0.2 \\
\hline 14 & 9 & 81.7 & 11.8 & 0.5 & 2.0 & 2.4 & 0.8 & 0.2 & 0.2 & 0.0 & 0.0 & 0.3 & 0.2 \\
\hline 15 & 2 & 67.6 & 24.8 & 0.0 & 0.7 & 3.5 & 0.6 & 0.6 & 1.3 & 0.0 & 0.0 & 0.7 & 0.3 \\
\hline 16 & 1 & 79.4 & 13.6 & 0.0 & 1.4 & 2.8 & 1.2 & 0.6 & 0.9 & 0.0 & 0.0 & 0.0 & 0.0 \\
\hline 17 & 5 & 79.9 & 10.5 & 1.1 & 2.2 & 2.8 & 2.3 & 0.4 & 0.4 & 0.2 & 0.0 & 0.3 & 0.1 \\
\hline 18 & 1 & 54.5 & 40.3 & 0.7 & 0.0 & 2.1 & 0.0 & 0.6 & 0.0 & 0.0 & 0.0 & 1.3 & 0.5 \\
\hline 22 & 1 & 78.9 & 13.6 & 0.0 & 4.3 & 1.4 & 1.2 & 0.6 & 0.0 & 0.0 & 0.0 & 0.0 & 0.0 \\
\hline 24 & 1 & 64.5 & 25.6 & 0.7 & 0.7 & 2.8 & 1.9 & 1.2 & 2.6 & 0.0 & 0.0 & 0.0 & 0.0 \\
\hline 25 & 1 & 79.1 & 15.8 & 0.0 & 0.0 & 2.1 & 1.9 & 0.6 & 0.0 & 0.0 & 0.0 & 0.0 & 0.5 \\
\hline 40 & 1 & 79.7 & 15.7 & 0.0 & 0.7 & 1.4 & 2.5 & 0.0 & 0.0 & 0.0 & 0.0 & 0.0 & 0.0 \\
\hline 47 & 1 & 60.1 & 32.1 & 0.0 & 3.7 & 2.9 & 0.6 & 0.6 & 0.0 & 0.0 & 0.0 & 0.0 & 0.0 \\
\hline
\end{tabular}


Table 7: Comparison of chemical compositions of bulk fly ashes.

\begin{tabular}{|c||c|c|c||c|c|c|}
\hline \multicolumn{1}{|c||}{} & \multicolumn{3}{c|}{ Upper Freeport } & \multicolumn{3}{c|}{ Kentucky \#9 } \\
\hline & CCSEM (1774) & Microprobe & PSI & CCSEM (950) & Microprobe & PSI \\
\hline $\mathrm{SiO}_{2}$ & 59.54 & 51.36 & 50.6 & 61.57 & 47.19 & 44.6 \\
\hline $\mathrm{Al}_{2} \mathrm{O}_{3}$ & 28.57 & 27.51 & 24.6 & 18.10 & 28.95 & 20.6 \\
\hline $\mathrm{Fe}_{2} \mathrm{O}_{3}$ & 4.36 & 13.05 & 13.5 & 5.83 & 12.58 & 22.4 \\
\hline $\mathrm{CaO}$ & 1.45 & 2.59 & 2.2 & 5.29 & 5.17 & 3.1 \\
\hline $\mathrm{MgO}$ & 0.32 & 0.22 & 1.4 & 0.09 & 0.15 & 1.1 \\
\hline $\mathrm{Na}_{2} \mathrm{O}$ & 0.37 & 0.53 & 0.3 & 0.28 & 2.27 & 0.9 \\
\hline $\mathrm{K}_{2} \mathrm{O}$ & 2.50 & 3.16 & 3.1 & 2.78 & 2.24 & 2.6 \\
\hline $\mathrm{Cl}$ & 0.17 & 0.00 & N.A. & 0.37 & 0.00 & N.A. \\
\hline $\mathrm{TiO}_{2}$ & 0.88 & 1.08 & 1.2 & 1.02 & 1.02 & 1.1 \\
\hline $\mathrm{SO}_{3}$ & 0.97 & 0.00 & 2.4 & 3.83 & 0.00 & 4.7 \\
\hline $\mathrm{P}_{2} \mathrm{O}_{5}$ & 0.13 & 0.40 & 0.1 & 0.10 & 0.25 & 0.1 \\
\hline $\mathrm{BaO}$ & 0.73 & 0.08 & N.A. & 0.74 & 0.12 & N.A. \\
\hline
\end{tabular}

Table 8: Comparison of chemical compositions of bulk fly ashes.

\begin{tabular}{|c||c|c|c||c|c|c|}
\hline \multicolumn{1}{|c||}{} & \multicolumn{3}{c|}{ Beulah } & \multicolumn{3}{c|}{ San Miguel } \\
\hline & CCSEM (1111) & Microprobe & PSI & CCEM (1558) & Microprobe & PSI \\
\hline $\mathrm{SiO}_{2}$ & 38.07 & 31.71 & 21.6 & 60.02 & 64.66 & 65.9 \\
\hline $\mathrm{Al}_{2} \mathrm{O}_{3}$ & 26.23 & 33.52 & 14.5 & 21.71 & 21.51 & 20.5 \\
\hline $\mathrm{Fe}_{2} \mathrm{O}_{3}$ & 3.14 & 16.89 & 12.2 & 0.78 & 2.75 & 1.9 \\
\hline $\mathrm{CaO}$ & 10.75 & 7.56 & 17.1 & 2.96 & 4.85 & 3.2 \\
\hline $\mathrm{MgO}$ & 3.37 & 3.37 & 4.2 & 0.02 & 0.10 & 0.5 \\
\hline $\mathrm{Na} \mathrm{O}_{2} \mathrm{O}$ & 3.44 & 6.68 & 6.2 & 2.42 & 3.34 & 2.5 \\
\hline $\mathrm{K} \mathrm{O}_{2} \mathrm{O}$ & 1.76 & 1.49 & 0.3 & 1.02 & 1.80 & 1.8 \\
\hline $\mathrm{Cl}$ & 1.4 & 0.02 & N.A. & 0.20 & 0.01 & N.A. \\
\hline $\mathrm{TiO}_{2}$ & 0.93 & 0.67 & 0.0 & 0.73 & 0.91 & 0.9 \\
\hline $\mathrm{SO}_{3}$ & 10.15 & 0.01 & 21.1 & 0.50 & 0.00 & 2.7 \\
\hline $\mathrm{P}_{2} \mathrm{O}_{5}$ & 0.17 & 0.39 & 0.0 & 0.02 & 0.03 & $<0.1$ \\
\hline $\mathrm{BaO}$ & 0.58 & 0.62 & N.A. & 0.54 & 0.12 & N.A. \\
\hline
\end{tabular}


Size Distribution of Fly Ash Plotted on Log-Probability Axes

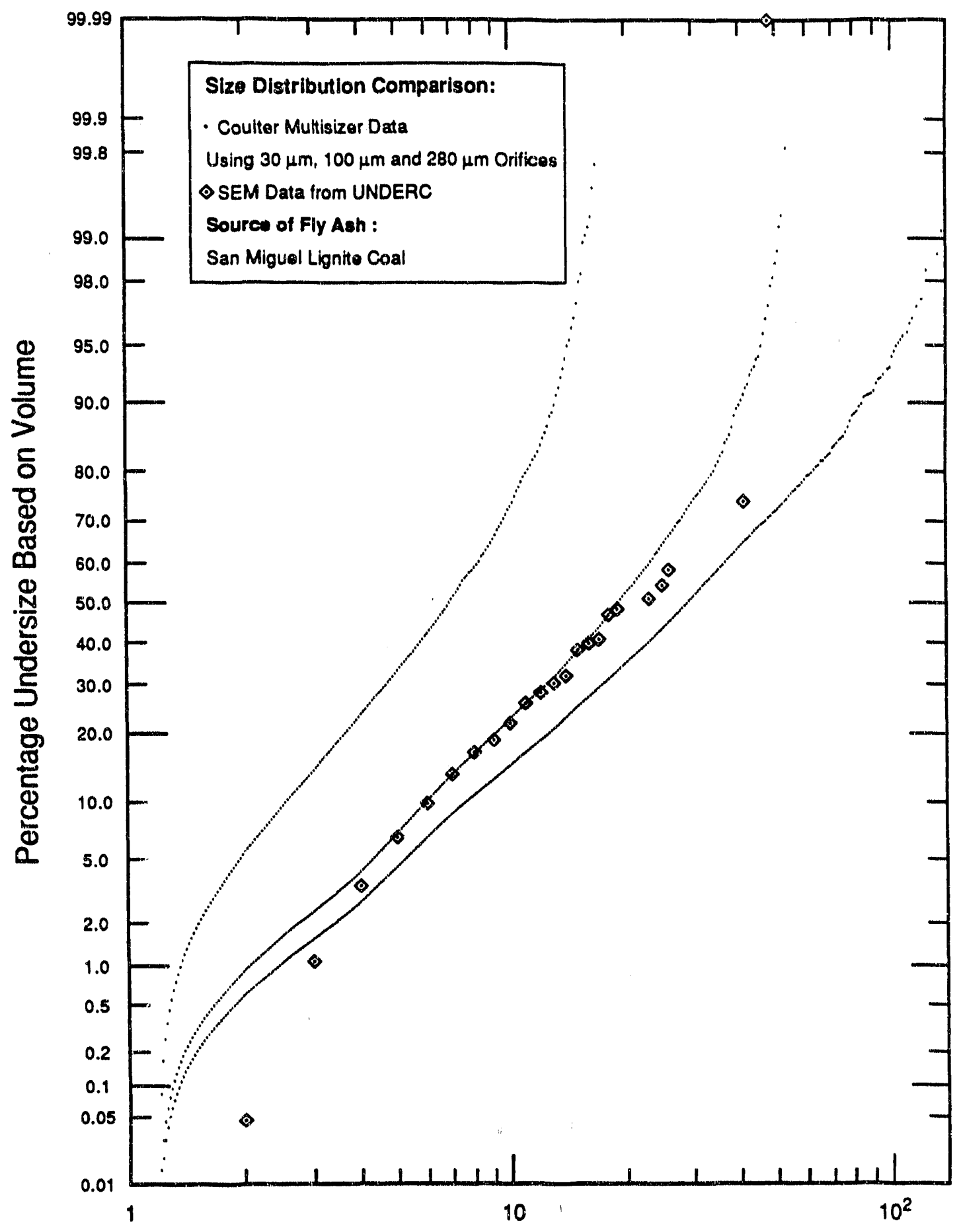

Log of Particle Diameter (in $\mu \mathrm{m})$

Figure 5: Comparison of San Miguel fly ash size distribution obtained by CCSEM and Coulter Multisizer 
Size Distribution of Fly Ash Plotted on Log.Probability Axes

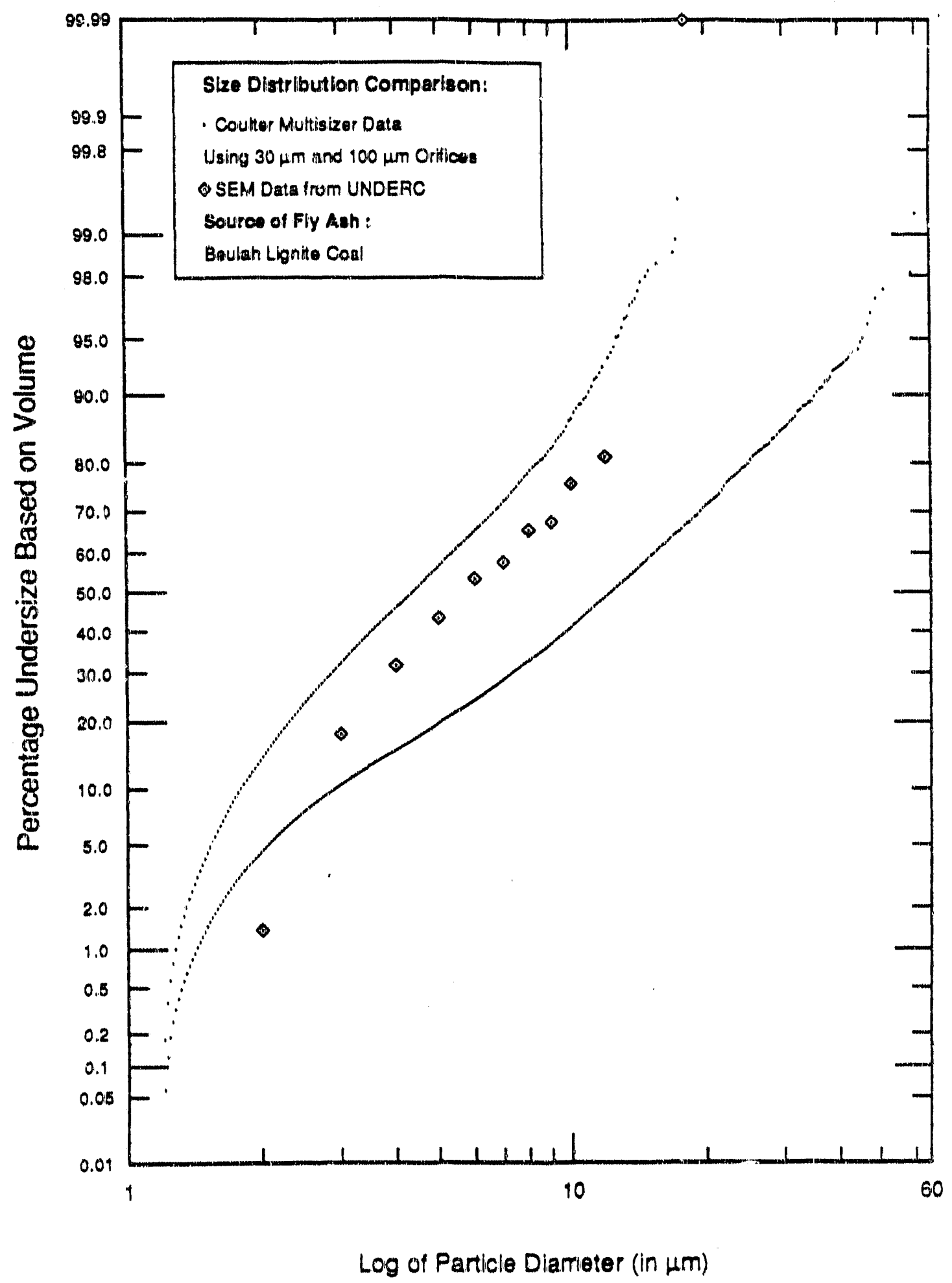

Figure 6: Comparison of Beulah fly ash size distribution obtained by CCSEM and Coultar? Mu!tisizar 
Size Distribution of Fly Ash Plotted on Log-Probability Axes

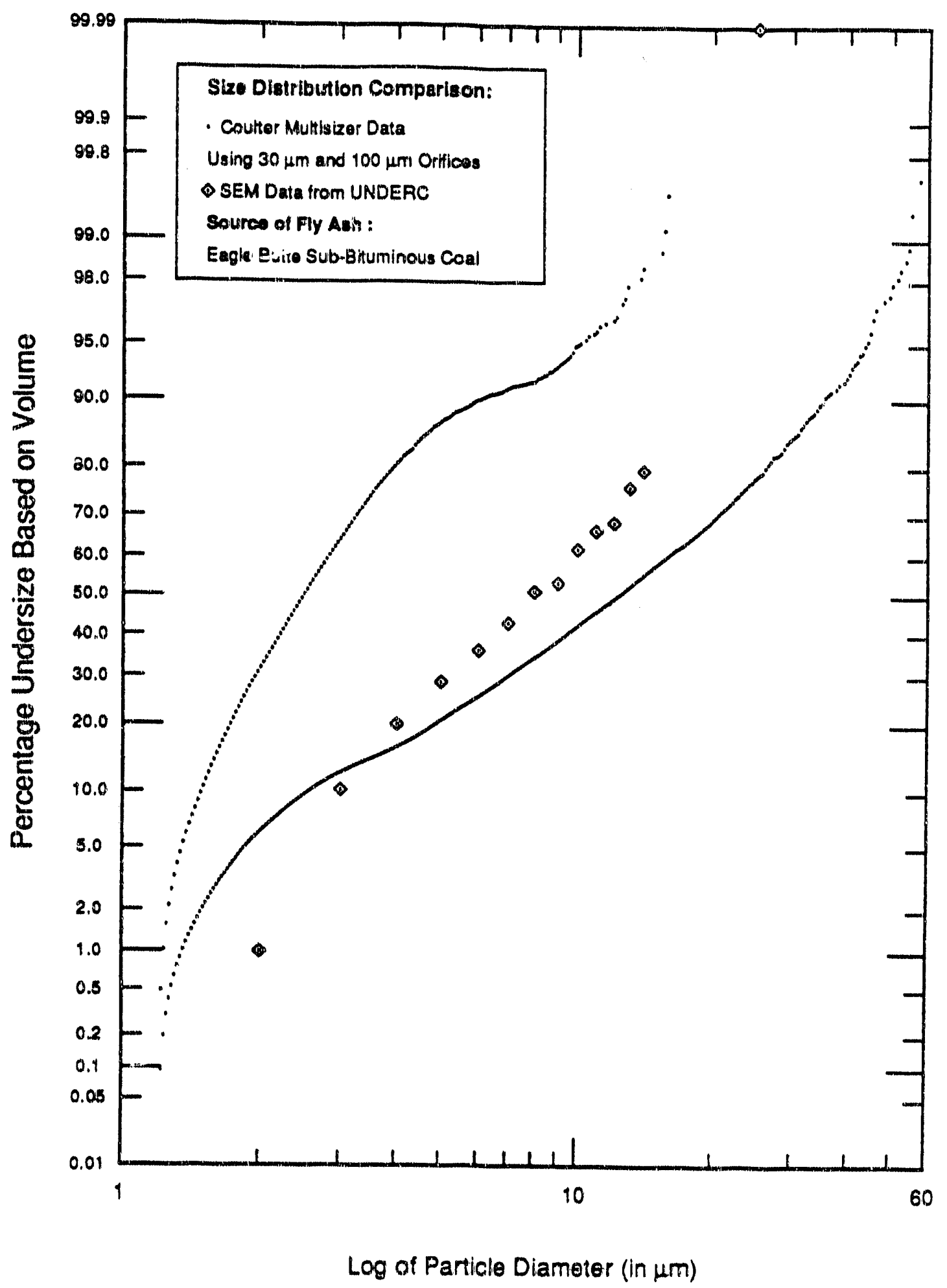

Figure 7: Comparison of Eagle Butte fly ash size distribution obtained by CCSEM and Coulter Multisizer 
Size Distribution of Fly Ash Plotted on Log-Probability Axes

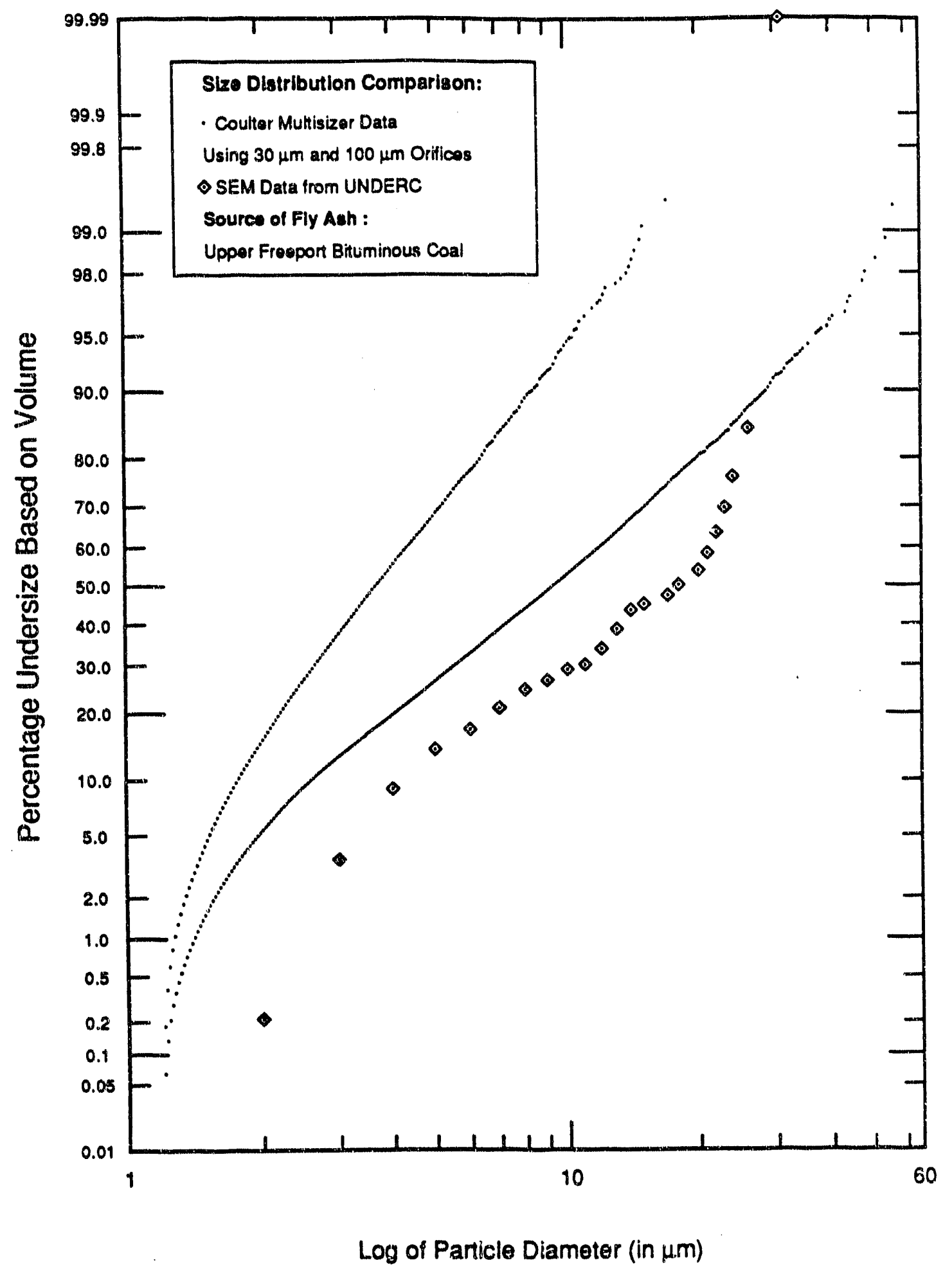

Figure 8: Comparison of Upper Freeport fly ash size distribution obtained by CCSEM and Coulter Multisizer 
Size Distribution of Fly Ash Plotted on Log-Probability Axes

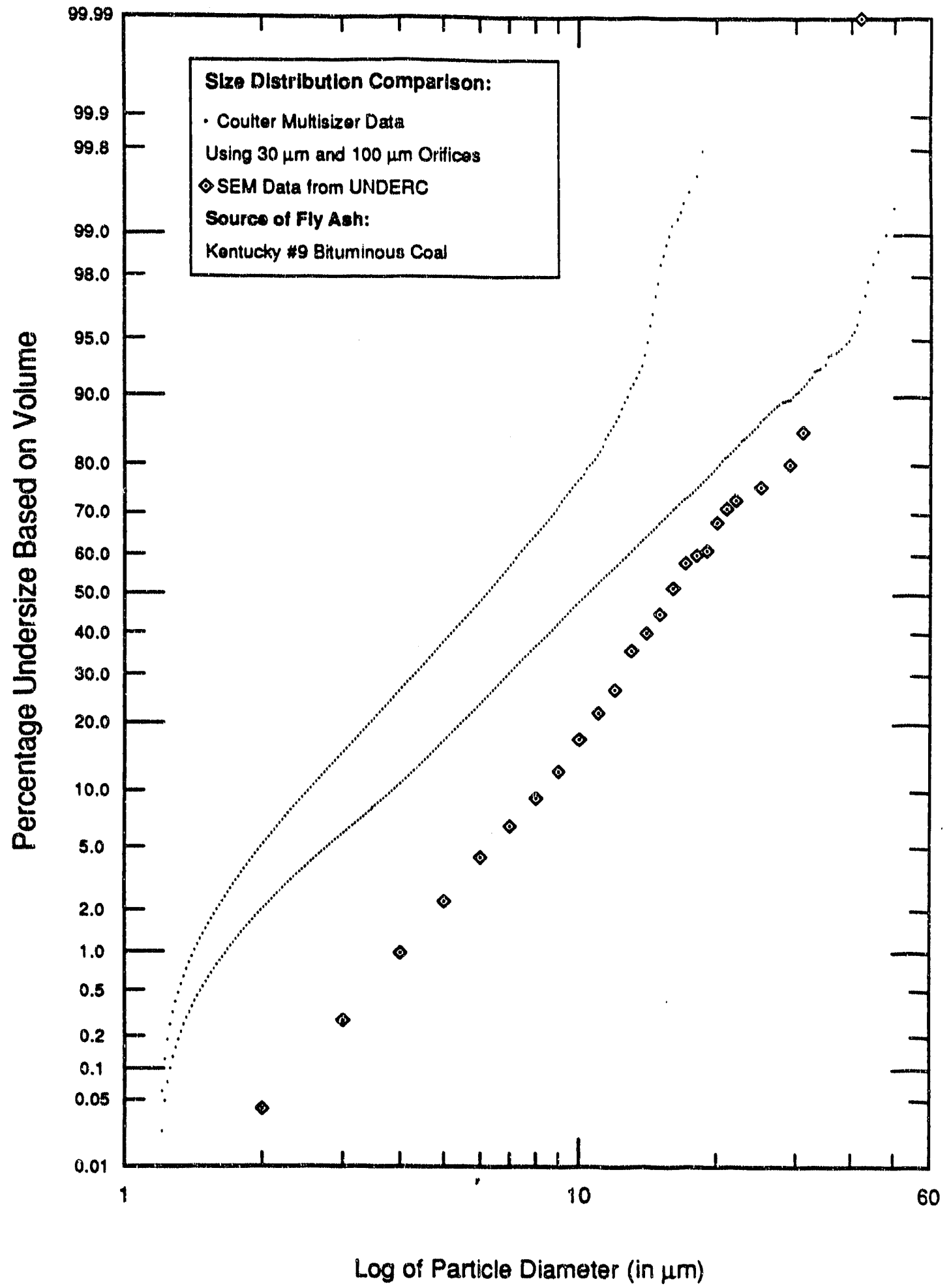

Figure 9: Comparison of Kentucky \#9 fly ash size distribution obtained by CCSEM and Coulter Multisizer 
Size Distribution of Fly Ash Plotted on Log-Probabillty Axes

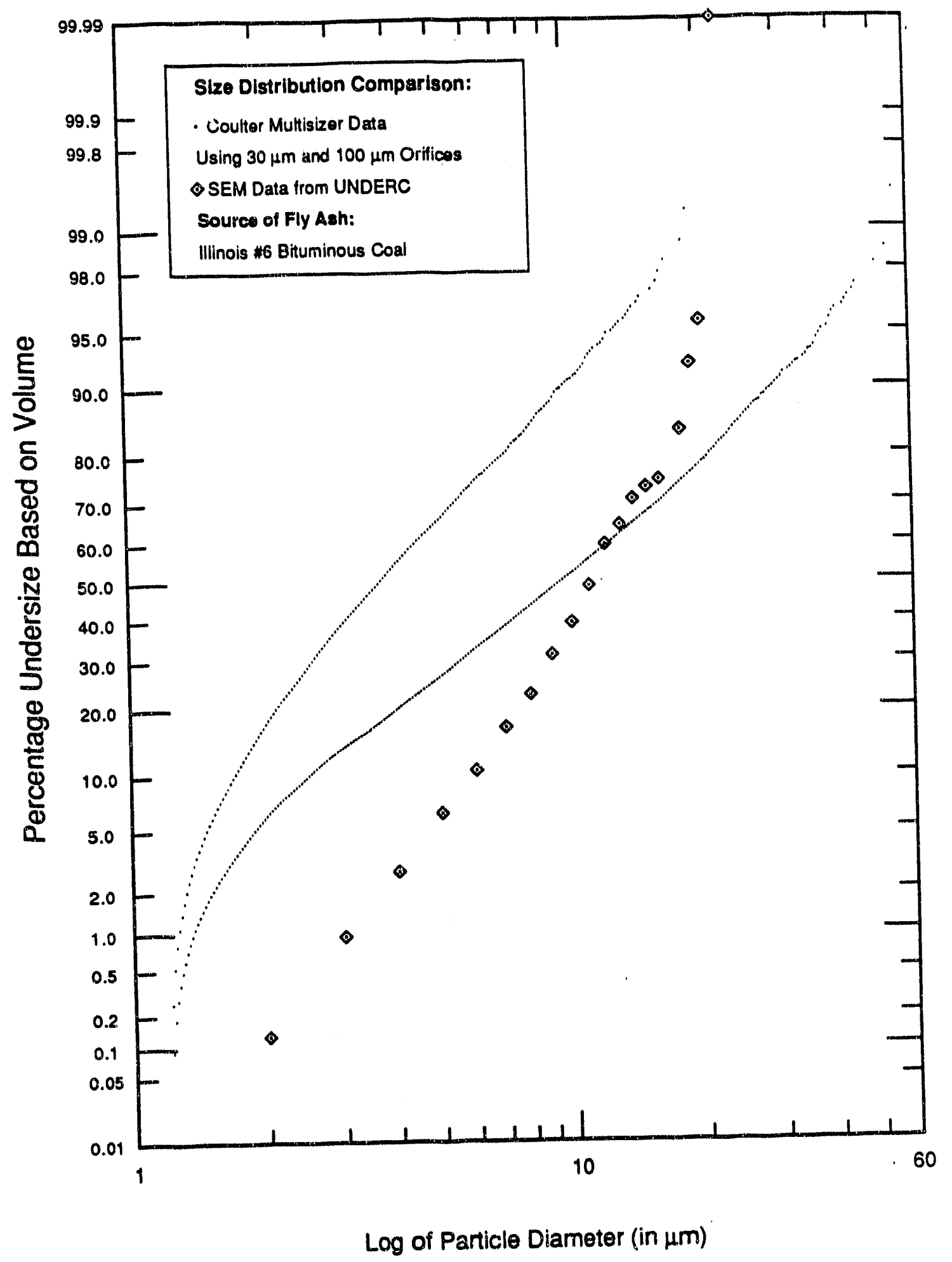

Figure 10: Comparison of lllinois \#6 fly ash size distribution obtained by CCSEM and Coulter Multisizer 
result, significantly fewer small fly ash particles are detected during the CCSEM. (See Tables 1-6). The median diameters by count, of all the ashes except San Miguel are smaller than $1 \mu \mathrm{m}$. However, the size distribution in the tables peak at some diameter between 1 and $2 \mu \mathrm{m}$. This explains the departure of the CCSEM curve from the Multisizer curve in the lower size range.

The difference in the two curves in the higher diameter ranges is due to statistical reasons. In the past QPRs the Multisizer data obtained using only the $30 \mu \mathrm{m}$ orifice was used to compare with the CCSEM size data because it was understood that ash particles of diameter only up to $20 \mu \mathrm{m}$ could be sized by the latter process. However, it now appears that the facility at UNDERC can size much larger particles. (The largest particle sized among the six samples has a diameter of $47 \mu \mathrm{m}$.) Hence, the CCSEM size data is now compared with the Multisizer data sized over the full range $(1.2 \mu \mathrm{m}$ to $>60 \mu \mathrm{m})$. Figure 5 shows the size distributions for San Miguel fly ash. The three Multisizer plots show, reading from left to right, the raw data obtained with (a) the $30 \mu \mathrm{m}$ orifice tube, (b) the combined data from the $30 \mu \mathrm{m}$ and the $100 \mu \mathrm{m}$ orifice tubes, and (c) the combined data from the $30 \mu \mathrm{m}, 100 \mu \mathrm{m}$ and the $280 \mu \mathrm{m}$ orifice tubes. Obviously, the median diameter from (a) is smaller than that obtained from the CCSEM data, since the $30 \mu \mathrm{m}$ orifice cannot size particles that are larger than $21 \mu \mathrm{m}$. However, it is seen that the complete size distribution (case (c)) yields a median diameter larger than that from the CCSEM data. This is due to the relatively small sample size of the latter (1558 particles) when compared with the Multisizer sample $(>200,000$ particles). The Multisizer sampled 23 particles of diameter $50 \mu \mathrm{m}$. Using this data, it is seen that approximately 4000 particles would have to be examined at random in the CCSEM study before a $50 \mu \mathrm{m}$ particle would be encountered. Again, some of these rare large particles could be rejected if they happened to be part of an agglomerate. As a result of sampling fewer large particles over the same size range as the Coulter Multisizer, the CCSEM size distribution is shifted to the left. It is not surprising that size distribution (b), which missed out the larger particles removed by the $53 \mu \mathrm{m}$ sieve, is quite close to the CCSEM distribution. The cases of Beulah (Figure 6), Eagle Butte (Figure 7), Upper Freeport (Figure 8), Kentucky \#9 (Figure 9) and Illinois \#6 (Figure 10) are very similar. In the last ash, the distortion in the size distribution due to the deficit of very small and very large particles has caused an anticlockwise rotation of the curve about the rnedian diameter. For the Kentucky \#9 ash, the effect of the deficit of larger particles is far less pronounced and the median diameter from the CCSEM data is about $40 \%$ larger than that of the Multisizer distribution.

With the exception of microprobe analysis of the two remaining slags (Eagle Butte and Illinois \#6) which will be performed this quarter, work on Task 2 on the physical and chemical characterization of the six fly ash samples, is now complete. However, the analysis of the data obtained will continue and will be reported in the next QPRs. 


\subsection{Task 2: The Optical Properties of High Temperature Slag}

The immediate goal of this task is to measure the high temperature optical properties of coal slag. The complex refractive index, $m=n+i k$, is being measured for synthetic slags at high temperatures, near $1900 \mathrm{~K}$. The effect of composition on the optical properties is also being investigated.

In the previous QPR's, measurements of the near normal reflectance of three different slag compositions were reported (SA00, SA01, and SA05). From those measurements the complex refractive index, $m$, was computed using the Kramers-Kronig relations, with $n$ being determined for the wavelength range $1<\lambda<13 \mu m$ and $k$ being determined for a narrower range $(8<\lambda<13 \mu \mathrm{m})$.

During the past quarter, two synthetic slags were made by melting mixtures of powdered oxides in an electric furnace at $1550^{\circ} \mathrm{C}$. Also, reflectance measurements for synthetic slags SA10 and SA20 (nominally 10 wt.\% and 20 wt. $\% \mathrm{Fe}_{2} \mathrm{O}_{3}$ respectively) were made.

\subsubsection{Selection of Slag Compositions}

The selection of the slag compositions is based on the criterion that the synthetic slags be representative of real fly ash with respect to its optical properties (or radiative properties). This criterion allows rejection of ash constituents that do not affect the optical properties.

The optical constants of slag in the wavelength range from 1-13 $\mu \mathrm{m}$ are primarily due to silica $\left(\mathrm{SiO}_{2}\right)$ and iron $\left(\mathrm{Fe}_{2} \mathrm{O}_{3}\right)$ in the slag, but few real ash particles are composed of just those two components. Most (by mass) real ash particles are derived from mirasals which contain substantial amounts of $\mathrm{Al}_{2} \mathrm{O}_{3}$ and/or $\mathrm{CaO}$ as well as many minor species.

Five slag compositions were proposed as a model for fly ash. These five slags (SA00, SA01, SA05, SA10, and SA20) are very similar except for iron content. Specifically, $\mathrm{SA} 00$ is nominally $58 \mathrm{wt} . \% \mathrm{SiO}_{2}, 29 \mathrm{wt} . \% \mathrm{Al}_{2} \mathrm{O}_{3}$, and $13 \mathrm{wt} . \% \mathrm{CaO}$ and SA $x x$ is SA00 
plus $x x$ wt. $\% \mathrm{Fe}_{2} \mathrm{O}_{3}$. This composition range reasonably spans the composition of most of the particles found in fly ash in so far as the optical constants of the material are concerned.

During the previous quarter computer controlled scanning electron microscope (CCSEM) results were obtained and analyzed (see Task 1) for various fly ashes. Based on these results, two new slags are now scheduled for optical properties measurement, in addition to the five SA $x$ slags for which the optical properties have already been measured. The basis for addition of the two new slags is presented below.

The Eagle Butte fly ash has a significantly different average composition than many other fly ashes. The Eagle Butte fly ash has lower levels of silicon and higher levels of calcium than do the five slags described above. Although $\mathrm{CaO}$ does not have an absorption mechanism in the near infrared (1-13 $\mu \mathrm{m})$, it is not known if the optical properties of this ash can be accurately modeled using the results from the higher silicon slags. Therefore, one of the new slags scheduled for measurement is a natural slag made from a melt of Eagle Butte fly ash.

Table 9 shows the averaged composition for the Eagle Butte ash from CCSEM results. The elemental measurements are converted to the oxides by assuming oxides as shown in previous Tables (Task 1). As discussed under Task 1, this average is obtained at each particle diameter, $D$, by averaging the composition of particles with diameter less than $D$. Since relatively few particles $(1,887)$ were analyzed, the presence of a few large particles can bias the results for the average of all particles. It is more instructive to look at the average composition for a specified diamter range of particles. For example, there is only one particle larger than $14 \mu \mathrm{m}$ and smaller than $25 \mu \mathrm{m}$ in the CCSEM results, but it changes the average silicon content from $21.4 \%$ to $25.0 \%$. Since particles larger than $25 \mu \mathrm{m}$ are not as important to radiative transfer in coal combustors as those smaller than $25 \mu \mathrm{m}$, one should, when possible, consider the composition as a function of particle size.

Since the natural slag made from Eagle Butte tly ash has many minor constituents, it is necessary to quantify their effect on the optical constants. The second new slag is a synthetic slag with composition similar to that of the natural Eagle Butte slag, but without the minor constituents. It is expected that measurements for this new synthetic slag will show the effect of the minor constituents. 
Table 9: Average Composition Analysis from CCSEM analysis of 1887 Particles of Eagle Butte Flyash. Averages (in Wt.\%) for Oxides (see Task 1) for particle diameters less than $D$ (in $\mu m$ ). N\% is percent by number undersized

\begin{tabular}{|c|c|c|c|c|c|c|c|c|c|c|c|c|c|}
\hline$D$ & $\mathrm{~N} \%$ & $\mathrm{Na}$ & $\mathrm{Mg}$ & $\mathrm{Al}$ & $\mathrm{Si}$ & $\mathrm{P}$ & $\mathrm{S}$ & $\mathrm{Cl}$ & $\mathrm{K}$ & $\mathrm{Ca}$ & $\mathrm{Fe}$ & $\mathrm{Ba}$ & $\mathrm{Ti}$ \\
\hline 2 & 35.612 & 0.4 & 9.3 & 25.0 & 19.3 & 0.7 & 4.3 & 0.4 & 0.3 & 34.7 & 3.1 & 1.0 & 1.6 \\
3 & 77.054 & 0.7 & 9.9 & 25.1 & 20.1 & 0.7 & 3.7 & 0.4 & 0.3 & 33.7 & 3.3 & 0.9 & 1.5 \\
4 & 90.037 & 0.8 & 9.9 & 25.0 & 19.3 & 0.7 & 3.6 & 0.3 & 0.3 & 34.5 & 3.4 & 0.9 & 1.4 \\
5 & 94.860 & 1.0 & 9.9 & 24.6 & 20.3 & 0.6 & 3.5 & 0.4 & 0.3 & 34.0 & 3.2 & 0.8 & 1.4 \\
6 & 96.979 & 1.0 & 10.7 & 24.7 & 19.7 & 0.6 & 3.4 & 0.3 & 0.4 & 34.0 & 3.2 & 0.8 & 1.3 \\
7 & 98.092 & 1.1 & 11.1 & 23.6 & 21.9 & 0.5 & 3.3 & 0.4 & 0.3 & 32.8 & 3.0 & 0.8 & 1.3 \\
8 & 98.940 & 1.1 & 10.5 & 22.5 & 23.4 & 0.5 & 3.1 & 0.4 & 0.3 & 33.3 & 3.0 & 0.8 & 1.4 \\
9 & 99.099 & 1.1 & 10.3 & 22.3 & 24.0 & 0.5 & 3.1 & 0.4 & 0.3 & 33.1 & 2.9 & 0.8 & 1.4 \\
10 & 99.523 & 1.0 & 9.6 & 22.4 & 25.1 & 0.5 & 3.0 & 0.4 & 0.3 & 32.9 & 2.8 & 0.8 & 1.4 \\
11 & 99.682 & 1.1 & 9.2 & 22.4 & 24.5 & 0.5 & 4.1 & 0.3 & 0.3 & 32.8 & 2.7 & 0.8 & 1.4 \\
12 & 99.735 & 1.0 & 9.0 & 21.9 & 24.0 & 0.5 & 4.1 & 0.4 & 0.3 & 33.8 & 2.8 & 0.9 & 1.5 \\
13 & 99.894 & 1.2 & 8.3 & 21.9 & 25.7 & 0.5 & 4.2 & 0.4 & 0.3 & 32.8 & 2.8 & 0.8 & 1.4 \\
14 & 99.947 & 1.2 & 8.2 & 21.3 & 25.7 & 0.4 & 4.3 & 0.4 & 0.3 & 33.2 & 2.8 & 0.8 & 1.4 \\
5 & 100.000 & 2.2 & 6.8 & 24.2 & 29.8 & 0.4 & 3.6 & 0.5 & 0.4 & 27.8 & 2.7 & 0.6 & 1.1 \\
\hline
\end{tabular}

\subsubsection{Melt preparation}

Preliminary melts using the Eagle Butte fly ash were very troublesome. The low melting point of this fly ash caused it to readily dissolve the alumina crucible in which it was melted. There are two options, either make the melt and measure the optical properties at a lower temperature or use a crucible that is not dissolved by the slag. If the measurements are made at lower temperature, interpretation of the results relative to the other slags could be complicated by any effect of temperature on the optical constants. Also, it is not certain that the lower temperature will eliminate the problem of the alumina dissolving into the slag. Therefore, the latter option was chosen and platinum crucibles were purchased for making melts of the natural slag from Eagle Butte fly ash and of the new synthetic slag.

The Eagle Butte slag was made during the previous quarter. A platinum crucible was filled with Eagle Butte fly ash and placed in the electric furnace at $1550^{\circ} \mathrm{C}$ for approximately eight hours. Since this single melt did not produce enough slag for making submerged reflector measurements (see previous QPRs) the crucible was filled once more and heated at $1550^{\circ} \mathrm{C}$ for eight hours. This procedure produced approximately $25 \mathrm{ml}$ of solid slag, enough for making the necessary measurements. 


\subsubsection{Reflectance Measurements}

The reflectance $(R)$ of the two synthetic slags, SA10 and SA20, was measured during the previous quarter. Each of the slags were placed in the electric furnace and heated to $1600^{\circ} \mathrm{C}$. The optical system was purged with nitrogen and the reflectance was measured. See past QPRs for details of the procedure. The measured results for all five synthetic slags is shown in Fig. 11.

The measurements for slags SA10 and SA20 showed the usual levels of noise which is probably due to beam steering in the region of large thermal gradients near the entrance to th: furnace. This noise is discussed in more detail in earlier QPRs.

One surprising result that will have to be further investigated is the inconsistency in the trend of SA05, relative to the other four synthetic slags. In the short wavelength range $(\lambda<8 \mu \mathrm{m})$, the slags show an increase in reflectance corresponding to an increase in $\mathrm{Fe}_{2} \mathrm{O}_{3}$ levels. This increase in $R$ results almost entirely from an increase in $n$. At wavelengths longer than $8 \mu \mathrm{m}$ the trend is not as consistent.

In the strong O-Si-O vibrational absorption peak centered near $10 \mu \mathrm{m}$ either the measured reflectance of the four slags SA00, SA01, SA10, and SA20 is high or the measured reflectance of SA05 is low. This presumption is based on an "expectation" that the beh ior of SA.05 should be monotonically correlated to the other four slags. Since the resuits from slag SA05 were the first to be obtained, it seems plausible that a lack of skill in aligning the optics at each wavelength led to low measured reflectances. It is known from experience that one must carefully align the optics, and that failure to do so leads to low reflectance measurements. In fact, most errors that one can make during the measurement produce low reflectance measurements, since maximizing the reference path signal is relatively easy compared to maximizing the sample path signal.

The Kramers-Kronig (KK) relations, described in a previous QPR, were used to compute the absorption index, $k$, and real refractive index, $n$. Since the reflectance is not strongly dependent on $k$ when $k<0.1$, the $\mathrm{KK}$ relations cannot be used to compute $k$ in the wavelength range $\lambda<8 \mu \mathrm{m}$. In contrast, the reflectance of the slags does depend strongly on $n$ for the entire range $1<\lambda<13 \mu m$, and the KK relations are used to compute $n$ over that entire wavelength range (note that the KK relations become equivalent to the Fresnel relations in the small $k$ limit). 


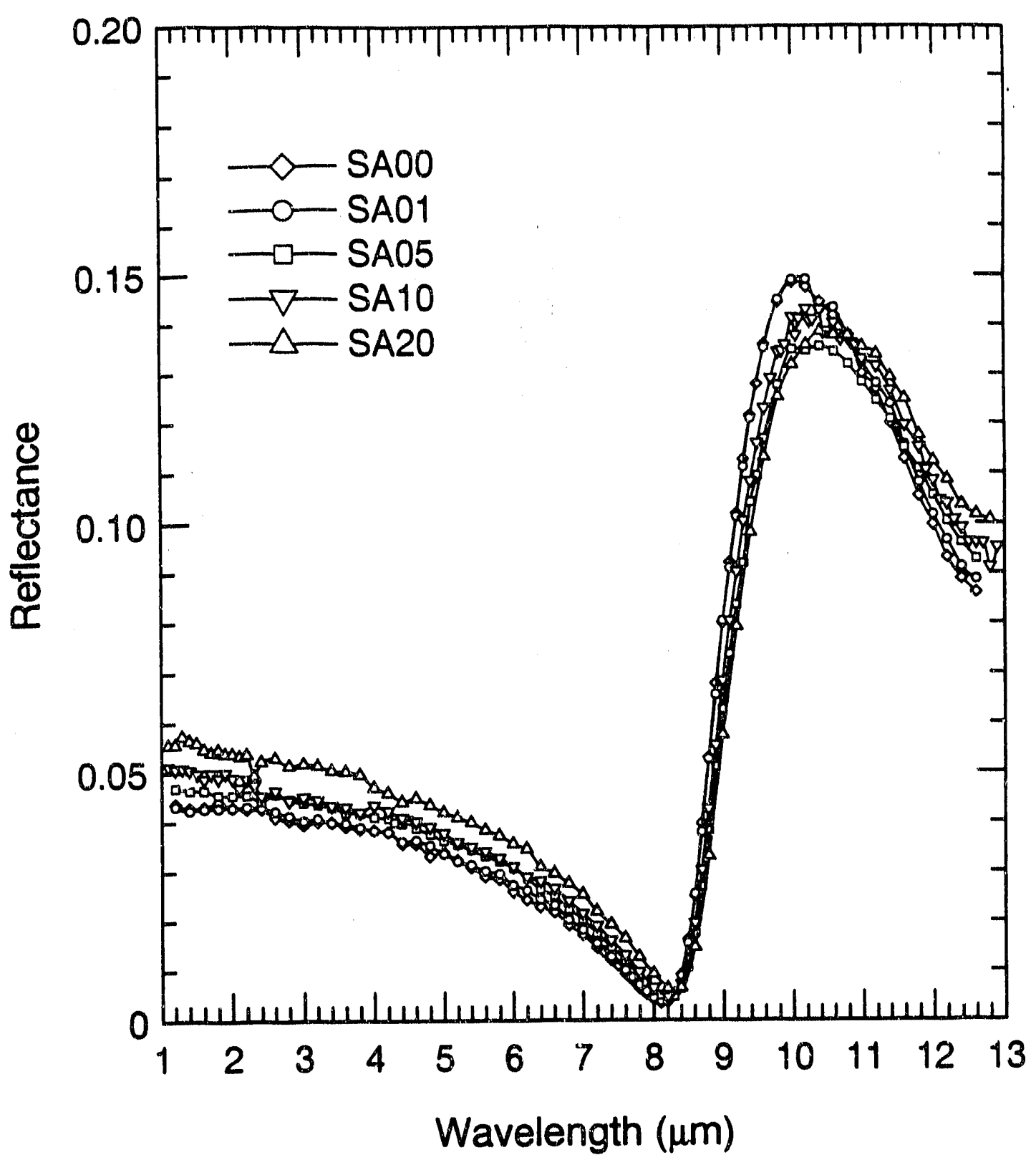

Figure 11: The reflectance of molten synthetic slags at $1600^{\circ} \mathrm{C}$

The absorption index, $k$, for the five synthetic slags is shown in Fig. 12. It is evident that $k$ does not vary significantly with composition in the range $8-13 \mu m$, at least not for the range of compositions studied here. Excepting SA05, for which we believe the measurements to be in error, the absorption band is broadened as the $\mathrm{SiO}_{2}$ concentrations are decreased (and the $\mathrm{Fe}_{2} \mathrm{O}_{3}$ is increased). The absorption index, $k$, 


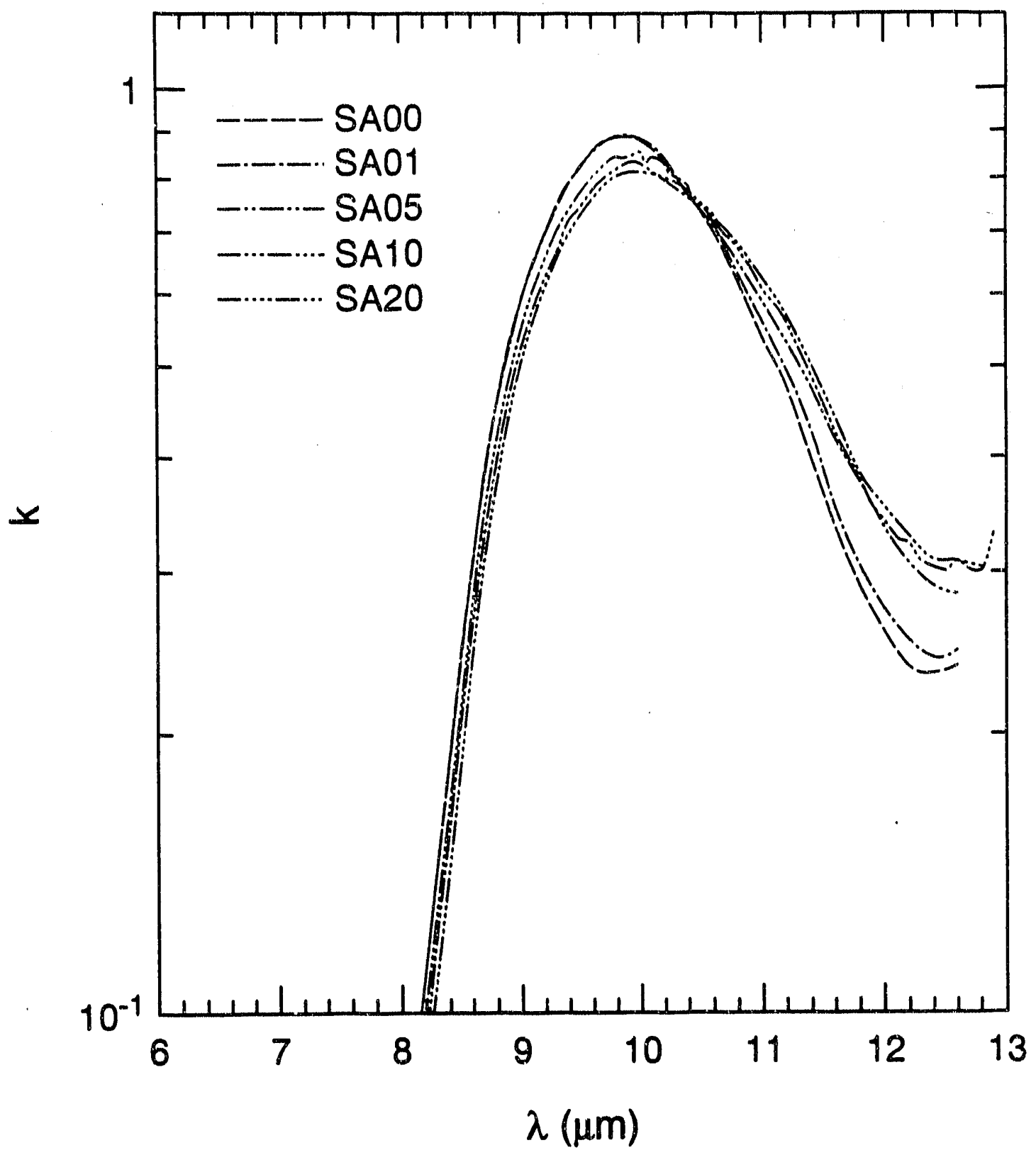

Figure 12: The absorption index of molten synthetic slags at $1600^{\circ} \mathrm{C}$

decreases near the peak at $10 \mu \mathrm{m}$ and increases near $12 \mu \mathrm{m}$ as the $\mathrm{Fe}_{2} \mathrm{O}_{3}$ levels are increased.

The real refractive index, $n$, is shown in Fig. 13 for the five synthetic slags. There is not a strong dependence of $n$ on the $\mathrm{Fe}_{2} \mathrm{O}_{3}$ concentration, but in the short wavelength range $(\lambda<8 \mu m) n$ increases somewhat with increased $\mathrm{Fe}_{2} \mathrm{O}_{3}$ concentrations. Near 


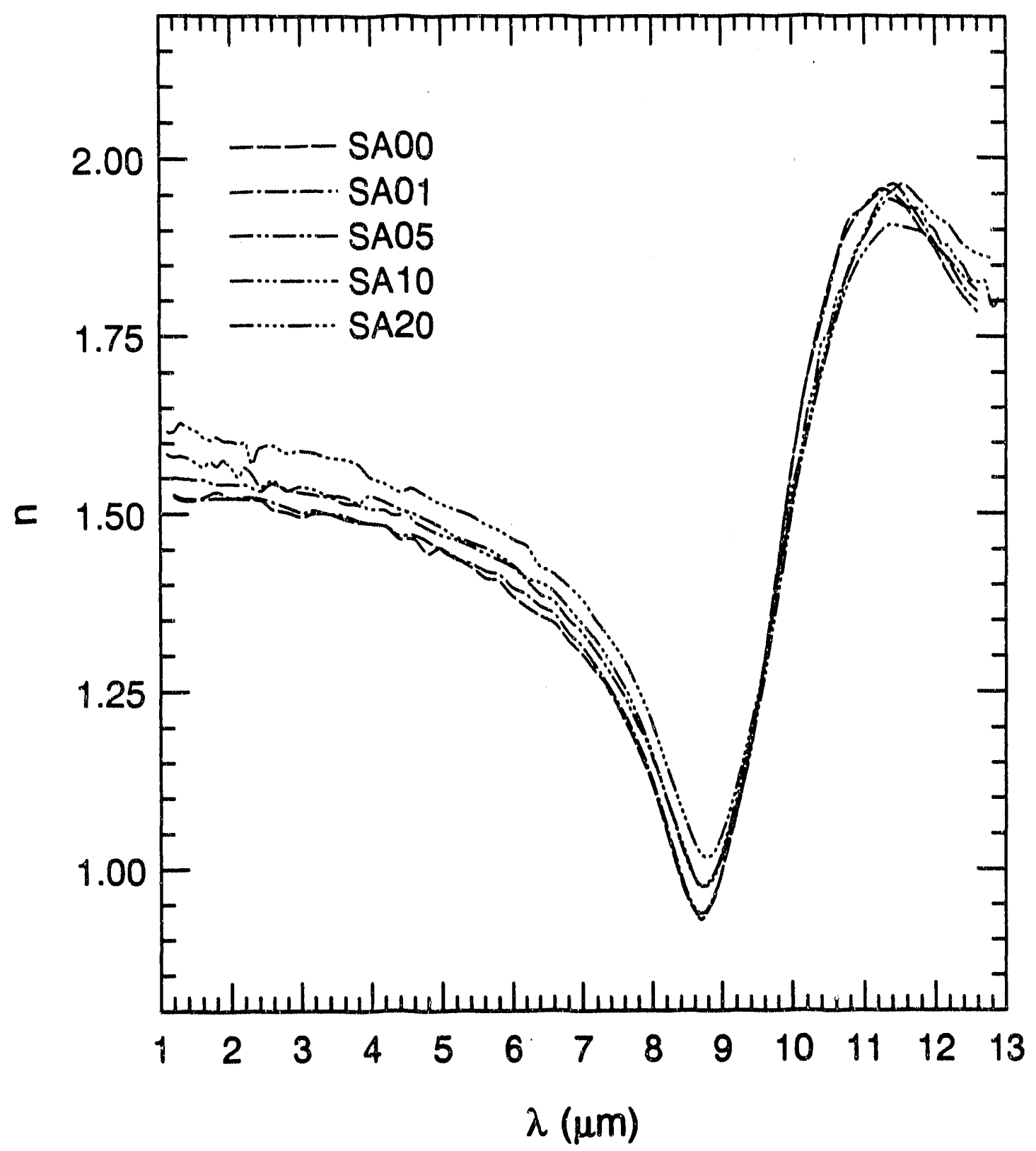

Figure 13: The real refractive index of molten synthetic slags at $1600^{\circ} \mathrm{C}$

$8.9 \mu \mathrm{m}$, where $n$ is a minimum, the increase in $\mathrm{Fe}_{2} \mathrm{O}_{3}$ levels causes a significant increase in $n$, a result that is consistent with the absorption band near $10 \mu \mathrm{m}$ being broadened. 


\subsubsection{Scheduled Activities}

During the next quarter measurements will continue with emphasis on completing all of the measurements, including those for the two new slags discussed above. Also, measurements will be carefully repeated until the unexpected trends iu the SA05 data are understood.

\subsection{Task 3: Sample Calculations of the Radiant Properties of Fly Ash Dis- persions}

During the previous quarter, several new computer codes were developed and previously developed codes were modified to allow investigation of the effects of fly ash on radiation heat transfer in coal combustion systems.

A flexible radiation transfer calculation technique is used to allow a study of the effects of anisotropic scattering and of temperature gradients in the medium. Also, this improved code allows one to consider non-uniform distributions of ash. During the past quarter several modifications to these codes provide accurate modeling of the scattering phase function. Example calculations were done using a simplified flyash model to evaluate the modifidcations.

\subsubsection{Overview}

In this research program, we have adopted the approach that by measuring fundamental properties (i.e, the complex refractive index, $m$ ) of the fly ash which participates in the radiation transfer, we can use well established theoretical principles (Mie theory) to compute the radiative properties of dispersions of fly ash as found in coal combustors. With this approach one can understand the underlying principles that affect the radiative properties of an ash dispersion and more confidently predict how variations in the characteristics of the ash dispersion cause variations in its radiative properties.

An important criterion in this approach is that the flyash particles be spherical, homogeneous, and isotropic. Fortunately, fly ash particles are formed at high temperatures at which most of them are molten, leading primarily to spherical particles. 
Furthermore, one should expect that molten particles will be reasonably homogeneous and isotropic. On cooling, most fly ash particles form glassy spheres which are homogeneous and isotropic, but some, and perhaps most from some types of coal, may form spheres or spheroids with interior bubbles or voids.

\subsubsection{Nonhomogeneous Particles}

While most flyash particles tend to form homogeneous solid spheres, there are some exceptional ashes that a mostly nonhomogeneous (i.e., having bubbles or voids). The San Miguel ash is apparently such an exception, since, based on density classification, most of the ash particles are not solid. See results under Task 1 for a detailed description.

There are some fortunate properties of these nonhomogeneous particles that partially relieve the modeler from the difficult task of aralyzing scattering and absorption by complicated geornetries. Since the ash particles are not solid, they tend to be very large (e.g. the volume median diameter of San Miguel ash is approximately $32 \mu \mathrm{m}$, more than twice that of the other ashes analyzed under Task 1). It is well understood that large particles (compared to the wavelength of radiation) do not effectively scatter radiation and can be ignored in most circumstances important to heat tranisfer in coal combustors. Furthermore, in the wavelength region where ash is strongly absorbing $(8-13 \mu \mathrm{m})$, one can ignore the voids and bubbles if most of the incident radiation is absorbed in a thin layer near the surface of the particle. For example, for $k=1.0$ and $\lambda=10 \mu \mathrm{m}$, approximately $70 \%$ of the radiation entering a large particle is absorbed within the first $1.2 \mu \mathrm{m}$ from the surface; therefore, the absorption efficiency could be reasonably approximated using geometric optics models. Since the size parameter of a $30 \mu \mathrm{m}$ particle is rather liarge, $x=\pi D / \lambda \sim 10$, these arguments based on geometric optics models are reasonably accurate, and one can easily anticipate the particle's scattering and absorptio a characteristics. See the previous QPR for a more detailed discussion of scattering by large spheres.

\subsubsection{Average Radiative Properties}

Fly ash particles vary in diameter over several orders of magnitude and the composition varies from particle to particle. Before radiation heat transfer calculations can 
be made for real fly ash dispersions, average scattering and absorption coefficients must be computed.

\section{The Log-Normal Particle Size Distribution}

The log normal size distribution accurately models the distribution of fly ash particle diameters, as reported under Task 1. In this model the particle size distribution function, $f(D)$, is defined so that $f(D) d D$ is the fraction of particles with diameter $D$ within $d D$. For a log-normal distribution

$$
f(D) \equiv \frac{1}{D \sqrt{2 \pi} \sigma_{u}} \exp \left(-\frac{(\ln D-\bar{u})^{2}}{2 \sigma_{u}^{2}}\right),
$$

where $\bar{u}=\ln \bar{D}$ and $\sigma_{u}=\ln \sigma_{g}$. The number median diameter is $\bar{D}$ (half the particles are smaller than $\bar{D}$ ) and $\sigma_{g}$ is the geometric standard deviation. It is more convenient to recast Eq. (1) in terms of $u=\ln D$ and write it as a differential function

$$
\frac{d f}{d u}=\frac{1}{\sqrt{2 \pi} \sigma_{u}} \exp \left(-(u-\bar{u})^{2} / 2 \sigma_{u}^{2}\right)
$$

where $d u=d(\ln D)=d D / D$. In this form we can easily interpret the distribution as a normal distribution centered about $\bar{u}$ with approximately $68.3 \%$ of the particles within $\pm \sigma_{u}$ of $\bar{u}$ and approximately $95.4 \%$ of the particles within $\pm 2 \sigma_{u}$ of $\bar{u}$. Also, the differential $d f$ is equal to $f d D$, which was interpreted above.

The $n^{i}$ moment of $d f$ is obtained by multiplying Eq. (2) by $D^{n}$ and normalizing so that the integral over all diameters is unity. If we adopt the notation where $d f_{n}$ is the $n^{\text {th }}$ moment, then

$$
\frac{d f_{n}}{d u}=\frac{1}{\sqrt{2 \pi} \sigma_{u}} \exp \left(-\frac{\left[u-\left(\bar{u}+n \sigma_{u}^{2}\right)\right]^{2}}{2 \sigma_{u}}\right) .
$$

For $n=0$ we obtain $d f_{0}=d f$, which is just the differential number distribution function described earlier. If $n=3$, then $d f_{3}$ is the differential volume distribution function where $d f_{3}=f_{3} d D$ is the fraction of the total particle volume due to particles with diameter $D$ within the range from $D$ to $D+d D$. The function $f_{3}$ is the volume distribution function. Similarly, $d f_{2}$ is the differential area distribution function and $d f_{1}$ is the differential diameter distribution function. It is apparent that $f=f_{0}$ is the "number distribution function", which is perhaps less arnbiguous than the term "size distribution function", although the the latter nomenclature is more popular. We will wo both terms interchangeably. 
It is clear from this analysis that the volume median diameter, $\overline{D_{3}}$, is related to $\bar{u}$ and $\sigma_{u}$ by the relation

$$
\overline{D_{3}}=\exp \left(\bar{u}+3 \sigma_{u}^{2}\right)
$$

where half of the total particle volume is due to particles with diameter smaller than $\overline{D_{3}}$. Similarly, the area median diameter, $\overline{D_{2}}$, is given by

$$
\overline{D_{2}}=\exp \left(\bar{u}+2 \sigma_{u}^{2}\right) \text {. }
$$

In general, the $n^{\text {th }}$ median diameter, $\overline{D_{n}}$, is given by the relation $\overline{D_{n}}=\exp \left(\bar{u}+n \sigma_{u}^{2}\right)$.

Another useful relation is the Sauter median diameter, defined as

$$
\begin{aligned}
D_{32} & \equiv \frac{\int_{0}^{\infty} D^{3} f(D) d D}{\int_{0}^{\infty} D^{2} f(D) d D} \\
& =\exp \left(\bar{u}+\frac{5}{2} \sigma_{u}^{2}\right)
\end{aligned}
$$

\section{The Log.Normal Distributions for Fly Ash}

The size distribution for a number of fly ashes was reported under Task 1, and the best log-normal fit parameters are summarized in Table 10. Corresponding plots of the area distribution functions are shown if Fig. 14. The symbols on the curves in Fig. 14 are located at $\overline{D_{2}}$ and at diameters on either side of $\overline{D_{2}}$ within which $68.3 \%\left( \pm \sigma_{u}\right)$ and $95.4 \%\left( \pm 2 \sigma_{u}\right)$ of the particle area is contained. For all but the San Miguel fly ash, which is largely composed of non-homogeneous particles with bubbles and voids, almost all of the particle area (either surface area or cross sectional area) is due to particles larger than $0.2 \mu \mathrm{m}$ and smaller than $50 \mu \mathrm{m}$. The volume median diameter is typically between $9 \mu \mathrm{m}$ and $15 \mu \mathrm{m}$, and the geometric standard deviation ranges from approximately 2.0 to 3.5. The dark symbols show ashes that are either not whole (cyclone separated) or contain a predominant fraction of nonhomogeneous particles (San Miguel). These ashes generally have a larger area median diarneter and probably do not represent most fly ashes found in coal fired furnaces. The whole ashes (shown as light symbols in Fig. 14) generally have an area median diameter of approximately $3 \mu \mathrm{m}$.

For purposes of evaluation of the computer codes developed during previous quarters, a model distribution (labeled distribution A) was chosen with $\overline{D_{3}}=9.0$ and 
$\sigma_{g}=2.0$. This distribution represents the lower limit in both $\overline{D_{3}}$ and $\sigma_{g}$ and should not be interpreted as the "best" choice for a particular ash. Distribution $A$ is also shown in Fig. 14. Comparing distribution $A$ with the distributions for real fly ashes, it most closely resembles the cyclone separated Illinois \#6 or Kentucky \#9 ashes, but has a $\overline{D_{2}}$ closer to the whole Kentucky \#9 ash or the cyclone separated Beulah ash.

Table 10: Log-Normal Distribution parameters of several fly ashes.

\begin{tabular}{|c|c|c|c|c|c|c|}
\hline Flyash & $\overline{D_{3}}(\mu \mathrm{m})$ & $\sigma_{g}$ & $\overline{D_{2}}(\mu \mathrm{m})$ & $\bar{u}$ & $\sigma_{u}$ & $D_{32}(\mu m)$ \\
\hline Kentucky \#9 Bag & 10.64 & 2.26 & 5.473 & -0.081 & 0.815 & 4.86 \\
Kentucky \#9 Cyc & 15.67 & 2.02 & 9.558 & 0.642 & 0.703 & 6.54 \\
Upper Freeport & 9.25 & 2.80 & 3.204 & -0.864 & 1.030 & 5.97 \\
Illinois \#6 Bag & 9.70 & 3.02 & 2.859 & -1.044 & 1.105 & 7.47 \\
Illinois \#6 Cyc & 14.72 & 2.14 & 8.251 & 0.407 & 0.761 & 6.38 \\
Beulah Bag & 12.80 & 2.95 & 3.971 & -0.696 & 1.082 & 9.30 \\
Beulah Cyc & 11.63 & 2.34 & 5.645 & -0.097 & 0.850 & 5.53 \\
Eagle Butte & 13.68 & 3.45 & 2.952 & -1.099 & 1.238 & 15.41 \\
San Miguel & 32.23 & 2.89 & 10.450 & 0.289 & 1.061 & 22.30 \\
\hline
\end{tabular}

\section{Uniform Composition Ash Model}

In addition to the distribution of particle sizes, one must also know the optical constants of the particles before average radiative properties can be computed. For purposes of evaluating the computer codes, we choose the optical constants at a specific wavelength, $\lambda=4.0 \mu \mathrm{m}$, for room temperature synthetic slag SA05. The complex refractive index is $m=n+i k=1.49+i 1.95 \times 10^{-4}$. We assume that all the particles of this model ash have the same $m$. Assuming that all ash particles have the same complex refractive index may not be be realistic, and will be evaluated in subsequent quarters. We also assume the dispersion is composed of spherical homogeneous particles, so that Mie theory may be used to compute absorption and scattering properties.

\section{Averaging radiative properties over a log-normal size distribution}

Once the diameter and complex refractive index, $m=n+i k$, are known for a spherical homogeneous particle, one can compute its scattering efficiency, $Q_{\mathbf{s}}(D)$, absorption efficiency, $\mathrm{Q}_{\mathrm{a}}(D)$, and scattering phase function $\Phi(\theta, D)$ using Mie theory. For radiation heat transfer problems, the incident radiation is assumed to be unpolarized (or randomly polarized). The scattering efficiency is the fraction of power incident on an 


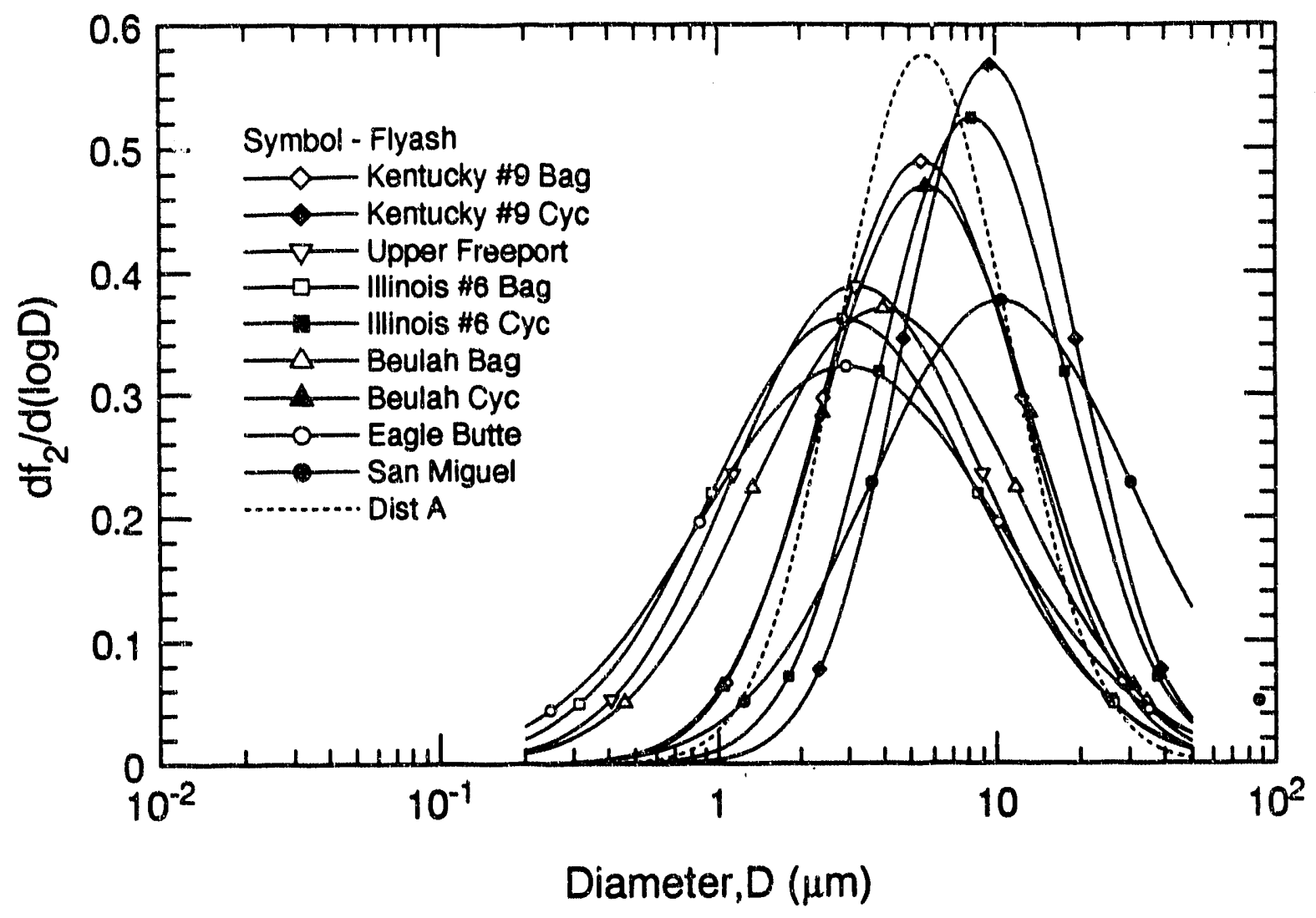

Figure 14: Area distribution functions for several fly ashes and model distribution A

area the size of the particle's cross section that is scattered, or redirected away from the direction of the incident beam. Similarly, the absorption efficiency is the fraction of power incident on the particle cross section that is absorbed by the particle. The scattering phase function describes the direction of the scattered light where $\theta$ is the scattering angle measured from the direction of incidence.

If the medium is composed of a dispersion of particles having a distribution of sizes described by a log-normal, then one can compute the average efficiencies and scattering 
phase function from the relations

$$
\begin{aligned}
\mathrm{Q}_{\mathbf{s}} & =\int_{-\infty}^{\infty} \mathrm{Q}_{\mathbf{s}}\left(e^{u}\right) f_{2}(u) d u \\
\mathrm{Q}_{\mathbf{a}} & =\int_{-\infty}^{\infty} \mathrm{Q}_{\mathbf{a}}\left(e^{u}\right) f_{2}(u) d u \\
\Phi(\theta) & =\frac{1}{\mathrm{Q}_{\mathbf{s}}} \int_{-\infty}^{\infty} \mathrm{Q}_{\mathbf{s}}\left(e^{u}\right) \Phi\left(\theta, e^{u}\right) f_{2}(u) d u
\end{aligned}
$$

Note that the lack of the argument $D$ in $\mathrm{Q}_{\mathrm{B}}, \mathrm{Q}_{\mathrm{a}}$, or $\Phi(\theta)$ denotes the size averaged (independent of $D$ ) properties. The equations above illustrate that the area distribution function is paramount in determining the radiative properties of a dispersion of particles. Particles with diameters at which $d f_{2} / d u$ (shown in Fig. 14) is small contribute little to the averaged radiative properties.

\subsubsection{Radiation Heat Transfer Calculations}

In the previous QPR we reported on a new computer code that was developed to compute the radiation heat transfer through a plane slab of anisotropically scattering particles. The code uses the discrete ordinates method to solve for the radiance and heat flux on a spatial grid in a finite number of directions (or ordinates). Several example calculations were presented for a monodispersion of particles (uniform size). Results indicated that an approximate model for the scattering phase function was needed since the complexity of the actual function makes computations expensive in terms of storage and execution time. A simple delta-function plus scaled isotropic scattering model for $\Phi(\theta)$ was presented and the error in that approximation was evaluated.

During the past quarier several improvements were made to the computer code discussed in the previous QPR. In particular, an alternative model for the scattering phase function was implemented and evaluated.

The $\delta-P_{N}$ approximation

The scattering phase function $\Phi(\theta)$ can be written as the Legendre series

$$
\Phi(\mu)=\sum_{j=0}^{\infty} A_{j} P_{j}(\mu)
$$

where $A_{0}=1$ and $\mu=\cos \theta$. In practice, the coefficients, $A_{j}$, become small for large $j$. For a spherical particle with size parameter $x$, the number of terms required to 
model the phase function using Eq. (8) is approximately $2 x+10$ [1-2]. This large number of terms translates into a requirement of roughly half the number of ordinate directions in the discrete ordinates solution method. Although this requirement is only moderately expensive for simple one dimensional problems, it causes a severe memory and performance burden when three dimensional problems are considered. Therefore, a simpler model for the scattering phase function is desirable.

As discussed in the previous QPR, the scattering phase function for a single particle is characterized by a large forward directed lobe (if the particle is moderately large) and a number (approximately $=x$ ) of smaller lobes spread approximately uniformly in $\theta$. As $x$ is increased the number of lobes increases and more terms in the Legendre series are required to describe the additional complexity. However, when the scattering is averaged over many particle sizes, as is the case for radiation heat transfer in an ash dispersion, the lobes vanish, and $\Phi(\theta)$ becomes a relatively smooth function in $\theta$. A plot of $\Phi(\theta)$ is shown in Fig. 15 for the model dispersion described above.

Although the complicated lobe structure in $\Phi(\theta)$ is removed by averaging over a distribution of particle sizes, the Legendre series still retains many significant terms. The averaged scattering phase function still has a strong forward scattering lobe that is not accurately modeled with a few orders of Legendre polynomials. However, if the forward lobe is modeled as a delta function then the remaining portion of the curve is reasonably modeled by only a few orders of Legendre polynomials. This approximation is the basis for the $\delta-P_{N}$ model.

Consider the phase function expansion [3]

$$
\Phi(\mu)=2 f \delta(1-\mu)+(1-f) \sum_{j=0}^{N} B_{j} P_{j}(\mu)
$$

where $f$ and $B_{i}$ are constants. As $N$ approaches infinity $f \rightarrow 0$ and Eq. (9) is equivalent to Eq. (8). Using Eq. (8) and the orthogonality relation

$$
\int_{-1}^{1} P_{j}(\mu) P_{i}(\mu) d \mu= \begin{cases}0, & \text { if } i \neq j \\ \frac{2}{2 j+1}, & \text { if } i=j\end{cases}
$$

the constants in Eq. (9) are determined in terms of the $A_{j}$ in Eq. (8), i.e.,

$$
f=\frac{A_{N+1}}{2 N+3} \text { and } B_{j}=\frac{A_{j}-(2 j+1) f}{1-f} .
$$

The approximate scattering phase function, Eq. (9), is shown in Fig. 15 for $N=1$, $N=5$, and $N=9$. The high accuracy of this $\delta-P_{N}$ model is evident for even relatively small $N$. 


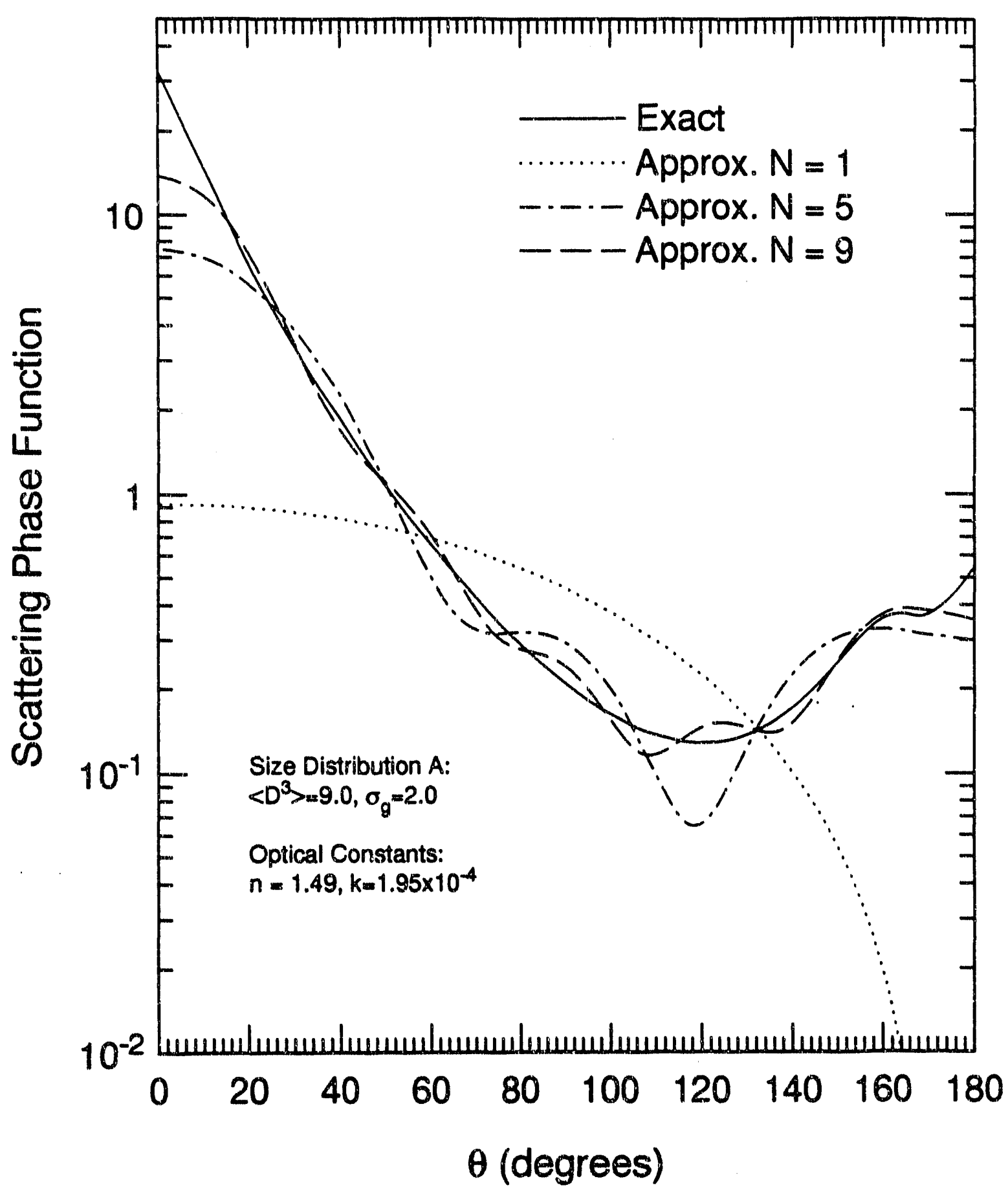

Figure 15: Scattering phase function, $\Phi(\theta)$ for the model particle distribution 
Evaluation of $\delta-P_{N}$ model for a planar slab

The $\delta-P_{N}$ model for $\Phi(\theta)$ was evaluated using the computer code developed during the past two quarters. A planar slab is assumed to have the particle size distribution and optical properties described previously. The scattering albedo, $\omega \equiv \mathrm{Q}_{\mathrm{s}} /\left(\mathrm{Q}_{\mathbf{s}}+\mathrm{Q}_{\mathrm{a}}\right)=$ 0.9979 , was computed from the size averaged scattering and absorption efficiencies.

The reflected and transmitted directional radiance is shown in Figs.16 and 17 for various optical depths, $\tau_{0}=\alpha L$, where the slab thickness is $L$ and the absorption coefficient is $\alpha$. The angle, $\theta$, on both plots is measured from the outward normal. We see that the error in the small $N$ approximations to $\Phi(\theta)$ is largest when $\tau_{0}$ is small. However, for $N=5$ or $N=9$ the error is very small for all $\tau_{o}$ shown.

The hemispherical transmittance, $T$, and reflectance, $R$, are shown in Table 11, along with the associated error for each phase function model. The results are consistent with Fig. 16 and Fig. 17 in that the error is largest for small $\tau_{o}$. However, it is significant that except when either $\mathrm{T}$ or $\mathrm{R}$ is small, even the $N=0$ solution is reasonably accurate. Furthermore, for $N=5$ the error is less than $0.55 \%$ for all the cases shown. Using $N=9$ gives essentially exact solutions for the hemispherical transmittance and reflectance.

From this analysis, it is evident that using the $\delta-P_{N}$ model for the scattering phase function is quite accurate for $N>5$. If errors on the order of a few percent can be tolerated, $N=2$ or less can be used. Also, if only the hemispherical fluxes are of interest, the $N=0$ solution is probably adequate. Since the computational expense of the $N=9$ solution is not high for a one dimensional problem, the $N=9$ approximation will be used for all further computations.

\subsubsection{Scheduled Activities}

The progress during the past two quarters has brought Task 3 near to completion apart from actually doing the calculations, which rely on data from Tasks 1 and 2 . Therefore, during the next quarter, efforts will be concentrated on completing the experimental measurements described under Task 2 and using the computer codes to guide the design of the ash dispersion measurements described under Task 4. 


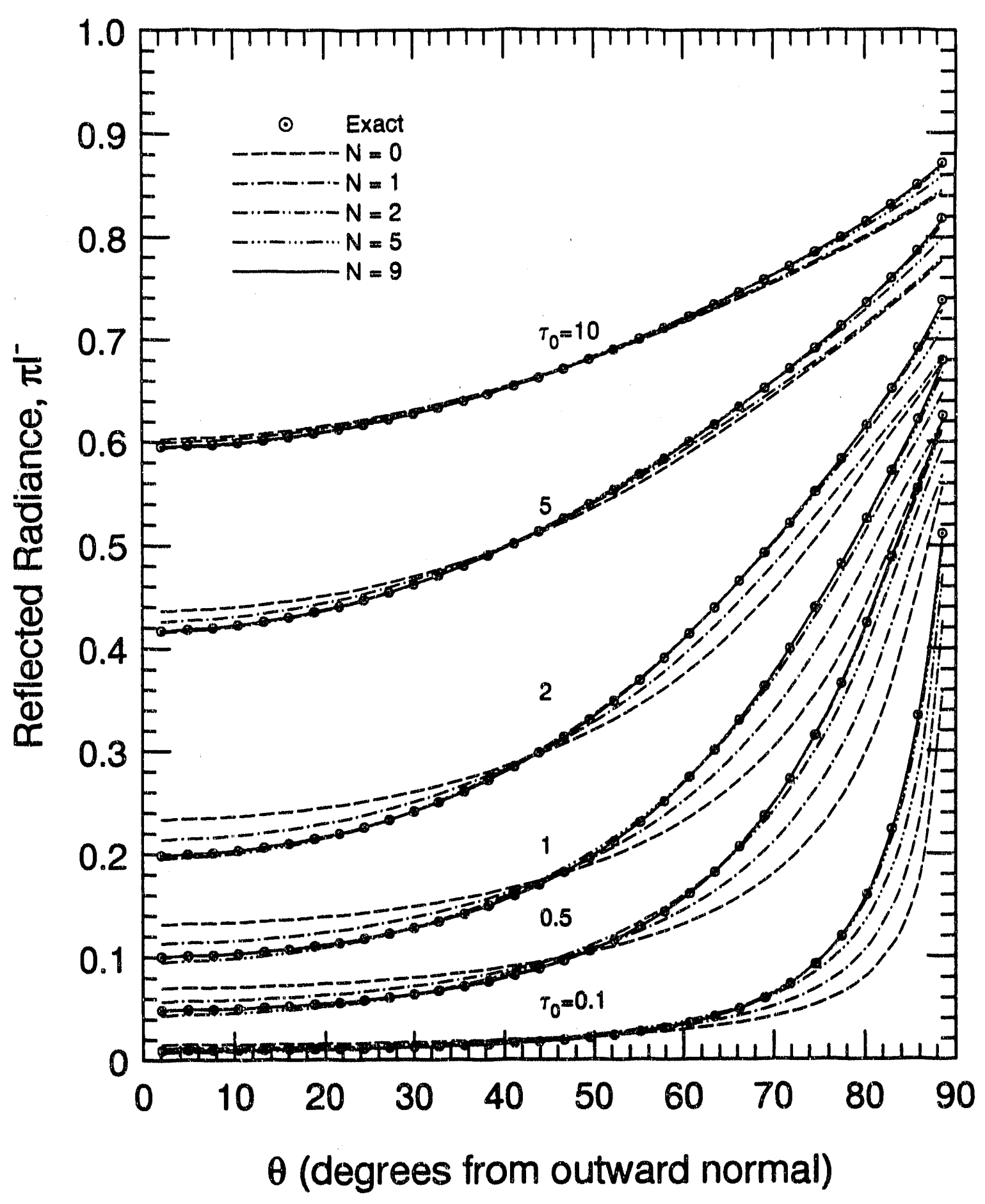

Figure 16: Reflected radiance for planar polydispersion with particle size distribution A, various optical depths, and $\delta-P_{N}$ model of $\Phi(\theta)$ with $N=0,1,2,5,9$ and $\infty$. 


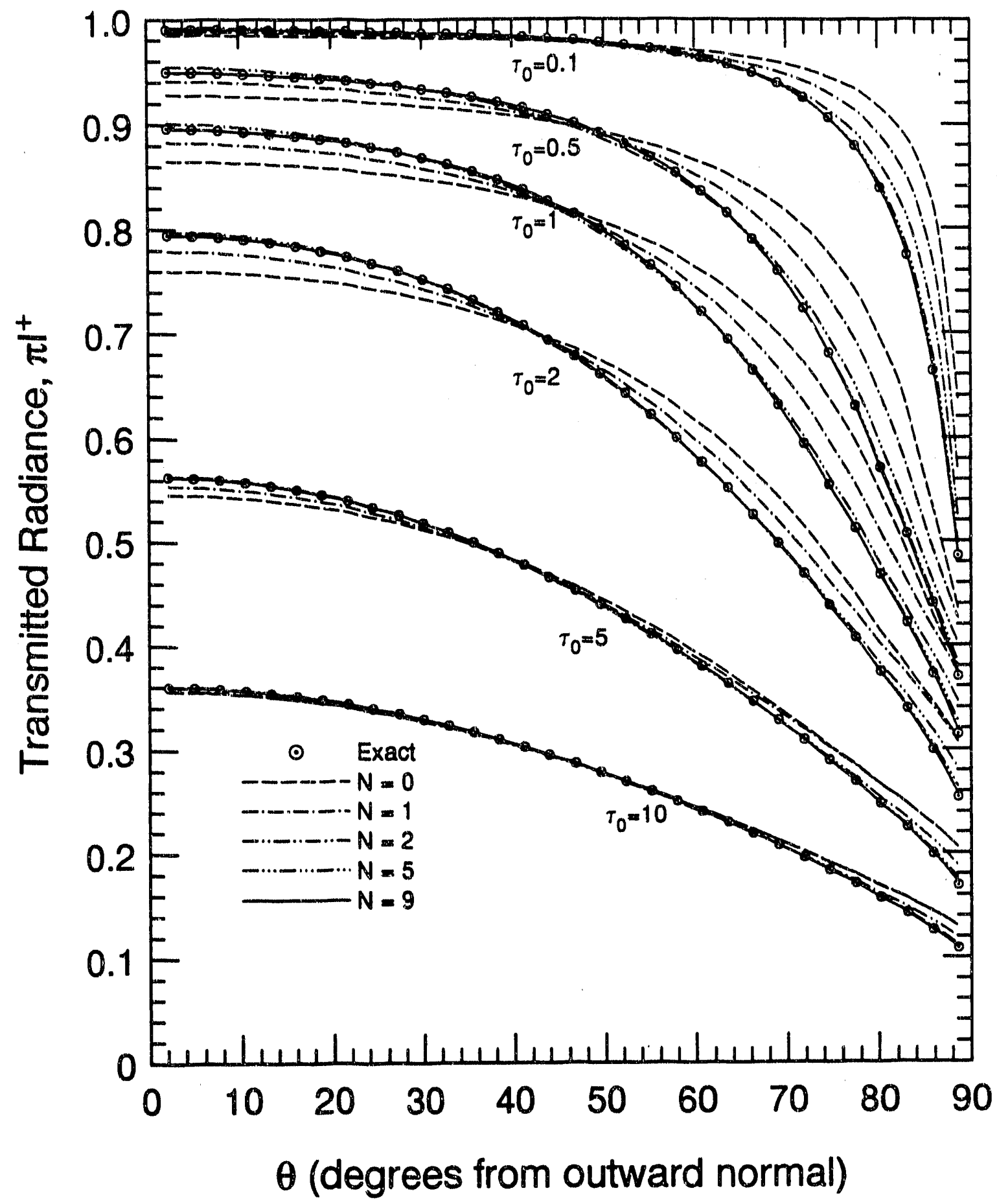

Figure 17: Transmitted Radiance for planar polydispersion with particle size distribution $\mathrm{A}$, various optical depths, and $\delta-P_{N}$ model of $\Phi(\theta)$ with $N=0,1,2,5,9$ and $\infty$. 
Table 11: Reflectance and Transmittance of Scattering Slab for Approximate Scattering Phase Function

\begin{tabular}{|c|c|c|c|c|}
\hline & \multicolumn{2}{|c|}{$\tau_{o}=0.1$} & \multicolumn{2}{c|}{ Percent Error } \\
\hline Order & $\mathrm{R}$ & $\mathrm{T}$ & $\mathrm{R}$ & $\mathrm{T}$ \\
\hline Exact & 0.03460 & 0.96499 & - & - \\
0 & 0.02810 & 0.971 .48 & -18.78 & 0.67 \\
1 & 0.03069 & 0.96889 & -11.29 & 0.40 \\
2 & 0.03337 & 0.96621 & -3.54 & 0.13 \\
5 & 0.03441 & 0.96518 & -0.55 & 0.02 \\
9 & 0.03457 & 0.96501 & -0.07 & 0.00 \\
\hline
\end{tabular}

\begin{tabular}{|c|c|c|c|c|}
\hline & \multicolumn{2}{|c|}{$\tau_{o}=0.5$} & \multicolumn{2}{c|}{ Percent Error } \\
\hline Order & $\mathrm{R}$ & $\mathrm{T}$ & $\mathrm{R}$ & $\mathrm{T}$ \\
\hline Exact & 0.13094 & 0.86699 & - & - \\
0 & 0.11943 & 0.87849 & -8.79 & 1.33 \\
1 & 0.12484 & 0.87308 & -4.66 & 0.70 \\
2 & 0.12993 & 0.86799 & -0.77 & 0.12 \\
5 & 0.13085 & 0.86707 & -0.07 & 0.01 \\
9 & 0.13093 & 0.86700 & -0.01 & 0.00 \\
\hline
\end{tabular}

\begin{tabular}{|c|c|c|c|c|}
\hline & \multicolumn{2}{|c|}{$\tau_{o}=1$} & \multicolumn{2}{c|}{ Percent Error } \\
\hline Order & $\mathrm{R}$ & $\mathrm{T}$ & $\mathrm{R}$ & $\mathrm{T}$ \\
\hline Exact & 0.21657 & 0.77928 & - & - \\
0 & 0.20633 & 0.78952 & -4.73 & 1.31 \\
1 & 0.21136 & 0.78449 & -2.41 & 0.67 \\
2 & 0.21601 & 0.77984 & -0.26 & 0.07 \\
5 & 0.21653 & 0.77933 & -0.02 & 0.01 \\
9 & 0.21657 & 0.77929 & -0.00 & 0.00 \\
\hline
\end{tabular}


Table 11: continued...

\begin{tabular}{|c|c|c|c|c|}
\hline & \multicolumn{2}{|c|}{$\tau_{o}=2$} & \multicolumn{2}{c|}{ Percent Error } \\
\hline Order & $\mathrm{R}$ & $\mathrm{T}$ & $\mathrm{R}$ & $\mathrm{T}$ \\
\hline Exact & 0.33752 & 0.65422 & - & - \\
0 & 0.33114 & 0.66060 & -1.89 & 0.97 \\
1 & 0.33420 & 0.65754 & -0.99 & 0.51 \\
2 & 0.33725 & 0.65449 & -0.08 & 0.04 \\
5 & 0.33749 & 0.65424 & -0.01 & 0.00 \\
9 & 0.33752 & 0.65422 & -0.00 & 0.00 \\
\hline
\end{tabular}

\begin{tabular}{|c|c|c|c|c|}
\hline & \multicolumn{2}{|c|}{$\tau_{0}=5$} & \multicolumn{2}{c|}{ Percent Error } \\
\hline Order & $\mathrm{R}$ & $\mathrm{T}$ & $\mathrm{R}$ & $\mathrm{T}$ \\
\hline Exact & 0.53624 & 0.44332 & - & - \\
0 & 0.53451 & 0.44505 & -0.32 & 0.39 \\
1 & 0.53507 & 0.44448 & -0.22 & 0.26 \\
2 & 0.53612 & 0.44344 & -0.02 & 0.03 \\
5 & 0.53622 & 0.44333 & -0.00 & 0.00 \\
9 & 0.53623 & 0.44332 & -0.00 & -0.00 \\
\hline
\end{tabular}

\begin{tabular}{|c|c|c|c|c|}
\hline & \multicolumn{2}{|c|}{$\tau_{o}=10$} & \multicolumn{2}{c|}{ Percent Error } \\
\hline Order & $\mathrm{R}$ & $\mathrm{T}$ & $\mathrm{R}$ & $\mathrm{T}$ \\
\hline Exact & 0.67825 & 0.28181 & - & - \\
0 & 0.67769 & 0.28236 & -0.08 & 0.20 \\
1 & 0.67776 & 0.28224 & -0.07 & 0.15 \\
2 & 0.67819 & 0.28185 & -0.01 & 0.01 \\
5 & 0.67823 & 0.28180 & -0.00 & -0.00 \\
9 & 0.67824 & 0.28179 & -0.00 & -0.01 \\
\hline
\end{tabular}




\subsection{Task 4: Measurement of the Radiant Properties of Fly Ash Dispersions:}

More tests have been conducted on the cell and further modifications have been made to the design. The experimental set-up is expected to be ready for making extinction measurements towards the end of this quarter. Details of the construction of the cell will be included in the next QPR. 


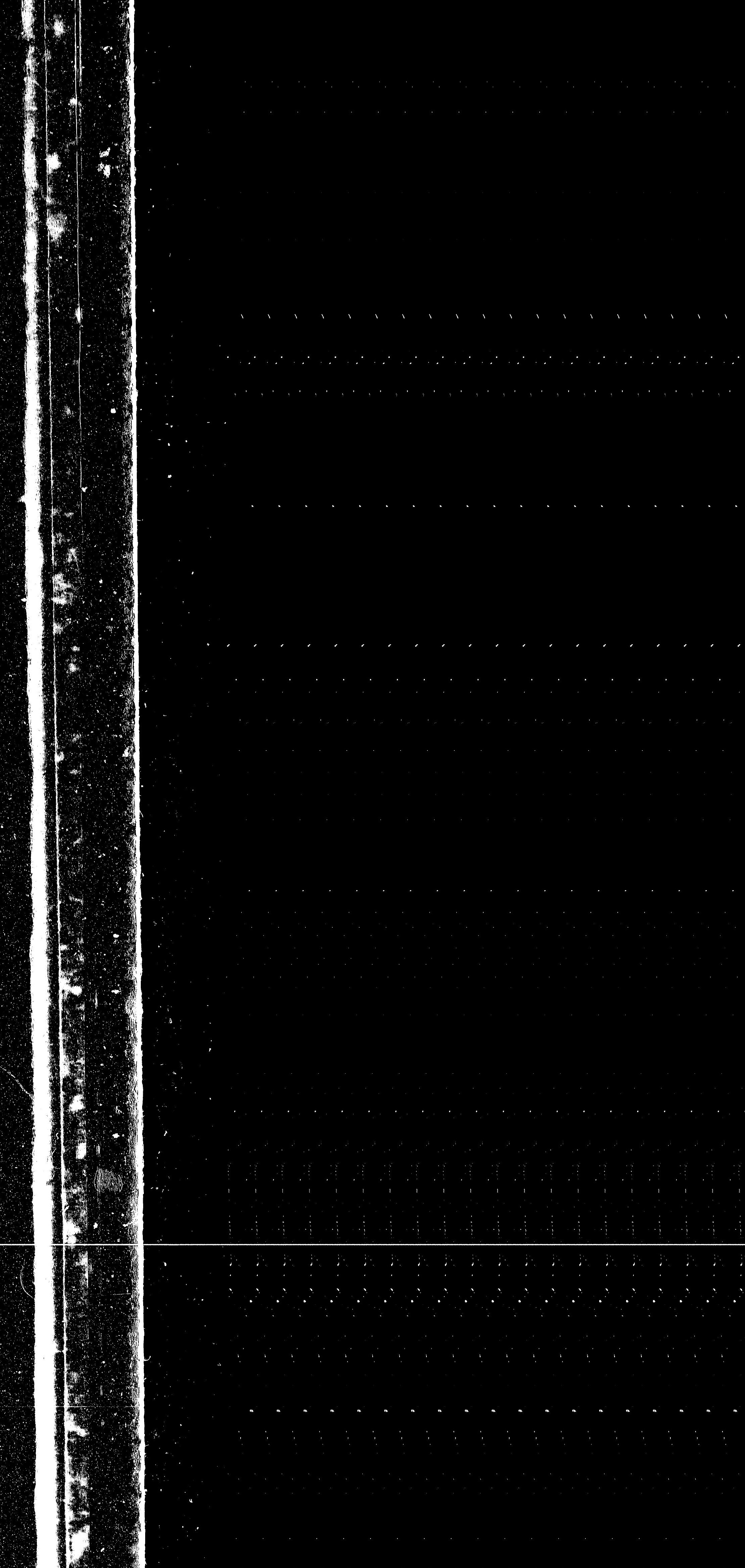




\subsection{REFERENCES}

1. PSI Technology Company, "Transformation of Inorganic Coal Constituents in Combustion Systems" Phase 1, Final Report Draft, March, 1990.

2. J. V. Dave, "Coefficients of the Legendre and Fourier series for the scattering functions of spherical particles," Applied Optics, Vol. 9, No. 8, 1970, pp. 1888-1898.

3. W. J. Wiscombe, "The Delta-M Method. Rapid Yet Accurate Radiative Flux Calculations for Strongly Asymmetric Phase Functions," Journal of Atmospheric Sciences, Vol. 33, 1976, pp. 2452-2459. 

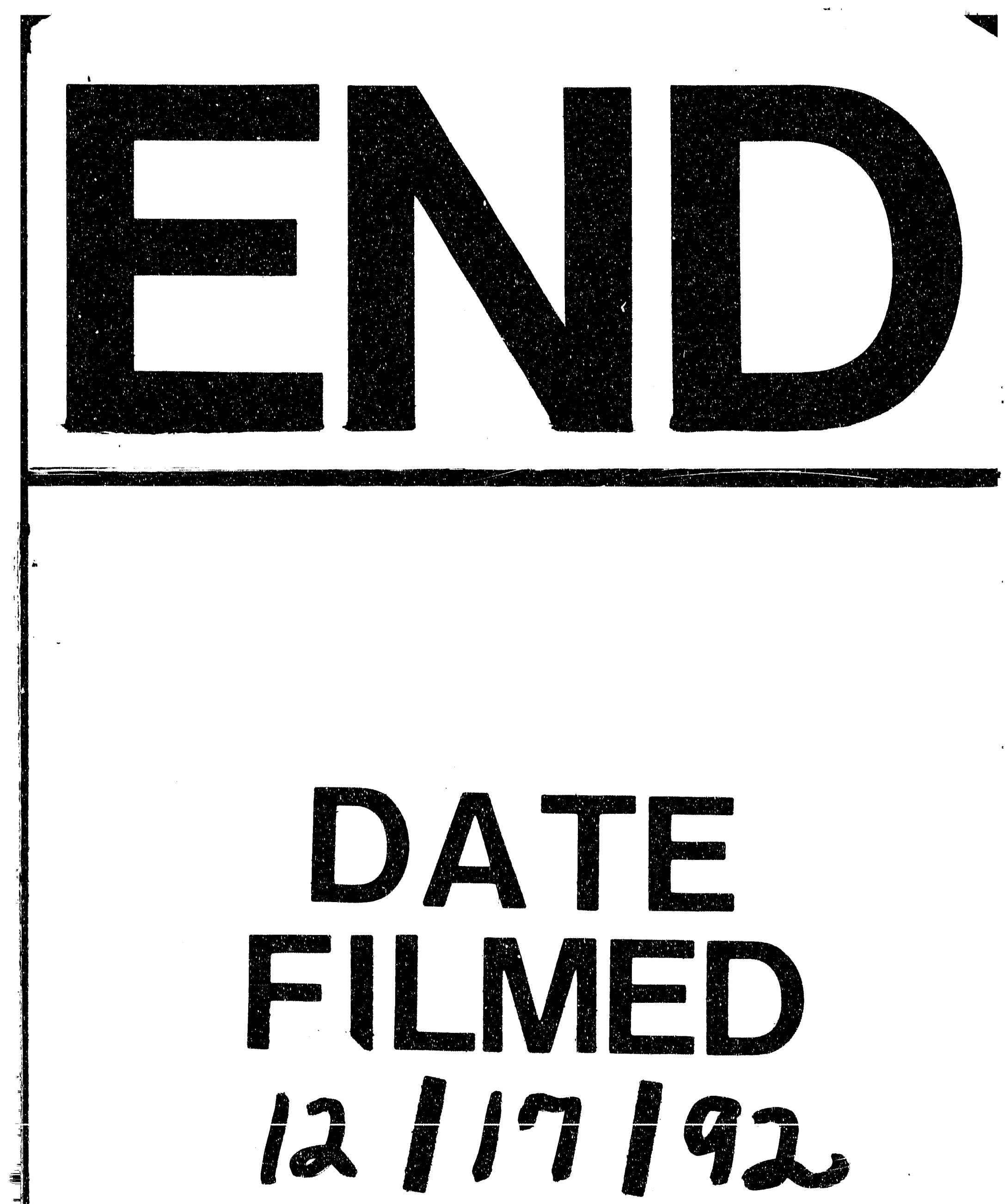


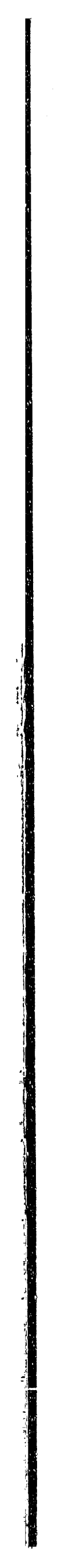

